\title{
Imaging evolution of Cascadia slow-slip event using high-rate GPS
}

3 Yuji Itoh $^{1 *}$, Yosuke Aoki ${ }^{1}$, Junichi Fukuda ${ }^{1}$

$5 \quad{ }^{1}$ Earthquake Research Institute, the University of Tokyo, Bunkyo-ku, Tokyo, 1136 0032, Japan.

*Corresponding author (yitoh@eri.u-tokyo.ac.jp)

\section{Abstract}

12 The slip history of short-term slow slip event (SSE) is typically inferred from daily Global

13 Positioning System (GPS) data, which, however, cannot image the sub-daily processes, leaving the 14 underlying mechanisms of SSEs elusive. To address the temporal resolution issue, we attempted to

15 employ the kinematic subdaily GPS analysis, which has never been applied to SSE studies because 16 its signal-to-noise ratio has been believed too low. By carefully post-processing sub-daily positions

17 to remove non-tectonic position fluctuation, our 30-minute kinematic data clearly exhibits the

18 transient motion of a few $\mathrm{mm}$ during one Cascadia SSE. A spatiotemporal slip image by inverting the

19 30-minute data exhibits a multi-stage evolution; it consists of an isotropic growth of SSE followed

20 by an along-strike migration and termination within the rheologically controlled down-dip width.

21 This transition at the slip growth mode is similar to the rupture growth of regular earthquakes,

22 implying the presence of common mechanical factors behind the two distinct slip phenomena. The

23 comparison with a slip inversion of the daily GPS demonstrates the current performance and

24 limitation of the subdaily data in the SSE detection and imaging.Better understanding of the non-

25 tectonic noise in the kinematic GPS analysis will further improve the temporal resolution of SSE. 


\section{Main body}

29 Various types of geophysical observations have captured the signatures of different types of slow

30 earthquakes, which are slow transient faulting with longer duration than regular fast earthquakes ${ }^{1-3}$. A

31 slow-slip event (SSE), a class of slow earthquake, is detected by geodetic observations ${ }^{4-15}$. It often

32 coincides with seismically detected slow earthquakes ${ }^{5-8,16-18}$, which are tremors, low-frequency

33 earthquakes (LFEs), and very low-frequency earthquakes. In particular, the term 'episodic tremor

34 and slip (ETS)' was coined to describe the coincidence of tremors and a slow slip ${ }^{16}$. With increasing

35 detection capability, slow earthquakes are now considered a common fault process in many

36 subduction zones around the world, such as Cascadia in northwestern US and western Canada ${ }^{1,4-12,}$

37 16-17 19-20, Mexico ${ }^{20-21}$, and Nankai and Tohoku in Japan ${ }^{2}, 13,18,22-23$, as well as continental transform

38 boundaries such as the San Andreas Fault zone ${ }^{24}$. They occur in a limited depth range, typically in

39 the deeper extension of the seismogenic zone of great regular earthquakes, due to the depth-

40 dependent change of rheological characteristics of fault zones ${ }^{1-3,20}$. The intermittent occurrence of

41 slow earthquakes adjacent to the seismogenic zone of great earthquakes has posed the question of

42 whether they trigger great earthquakes, which is an important issue in a social context ${ }^{2}$. Hence, the

43 underlying physical mechanisms of these slow earthquakes have been studied extensively with a

44 variety of mechanisms proposed ${ }^{3,26-27}$.

45 Global Navigation Satellite System (GNSS) is one of the most powerful tools for capturing surface

46 deformation associated with $\mathrm{SSE}^{4-15,20-21,23}$. Continuous GNSS position time series have been used

47 to detect short-term SSEs lasting a few to tens of days ${ }^{1-2}$. Simple temporally averaged fault models or

48 spatiotemporal evolution of SSE have been constructed from the extracted signature, providing

49 observational constraints to diverse physical models of slow earthquakes ${ }^{4-13,15}$. However, their

50 typical epoch-to-epoch interval is one day, hiding detailed slip processes of short-term SSEs with a

51 time scale less than a day or the sub-daily processes. The paucity of subdaily resolution of data needs

52 overcoming to understand the mechanisms of short-term SSEs and their physical relationship with

53 the seismically detected slow earthquakes with a much shorter duration of up to hundreds of

54 seconds ${ }^{1-3,16-18}$. Recent studies used tremors and LFEs as a proxy for small slips and suggested the

55 presence of subdaily slow slip activities ${ }^{15,20-21,23,28}$, so robust geodetic measurement techniques to

56 reveal the slip process in the sub-daily time scale has been awaited. While strainmetres and tiltmetres

57 are sensitive to the sub-daily time scale ${ }^{29}$ they are not as prevalent as GNSS in many plate boundary

58 zones, and their data suffer from instrumental drifts and local phenomena around the sites such as

59 groundwater flow and raining ${ }^{29}$. These features make it difficult to extract sub-daily SSE signatures

60 with them.

61 Daily static GNSS positions are deduced from a stack of raw observables of GNSS, which are the

62 carrier phase of microwaves typically recorded at 1-, 15-, or 30-second intervals, by assuming no site

63 motion during each day. In contrast, the kinematic GNSS analysis is a method to determine the

64 position of sites at every raw observation epoch ${ }^{30}$, so it has a large potential to overcome the

65 sampling interval issue of GNSS positions for short-term SSE studies. However, the typical noise 
66 level of the kinematic GNSS positions is in the order of centimetres ${ }^{30-32}$, larger than the typical short-

67 term SSE signature ${ }^{4-13}$; this has prevented its application in SSE studies. With careful post-

68 processing of kinematic positions, recent studies have successfully extracted sub-centimetre

69 postseismic deformation ${ }^{31-32}$, another class of slow tectonic deformation, using kinematic GNSS

70 positions. Motivated by these postseismic deformation studies, we attempted to apply kinematic

71 GNSS positioning to an SSE in Cascadia. This study presents the first application of kinematic GPS

72 to detect and image the SSE evolution. In this study, we processed raw GPS observables recorded

73 during a previously reported SSE in northern Cascadia from March to April $2017^{9}$ and inferred its

74 spatiotemporal slip from these data. Then, we discussed tectonic implications from the inferred SSE

75 process using the kinematic GPS. For the comparison purpose, we also derived a slip model of the

76 same event using the daily static coordinates and discussed the performance of the kinematic GPS

77 processing on the SSE exploration as well as its current limitation.

\section{Data analysis}

80 The ETS activity in northern Cascadia is well known to occur beneath the onshore area (Figure 1)

81 where the GNSS network has been deployed (Figures 2 and S1 - S3) ${ }^{4-12}$. An inversion of the daily

82 GPS data has revealed that the March to April 2017 Cascadia SSE migrated from $\sim 48.0 \mathrm{~N}$ towards

83 the northwest along the fault-strike with roughly concordant migration of tremors ${ }^{9}$. We obtained

84 kinematic subdaily positions at sites in northern Cascadia by processing the raw GPS observables at

85 an interval of 30 seconds and subsequently performed post-processing to retrieve the transient

86 deformation signature likely due to the SSE. By correcting the 30-seconds kinematic coordinates for

87 temporally ${ }^{33-35}$ and spatially ${ }^{36}$ correlated errors as well as some outliers, we finally obtained 30-

88 minute interval coordinate series at 64 sites (See Method) (Figure S3). The nominal error of these

89 coordinates at each epoch is typically $1-2 \mathrm{~cm}$ for the horizontal components. Yet, at most sites, our

90 cleaned 30-minute coordinates reproduced sufficiently the trajectory of the daily static coordinates

91 (Figures 2 and S1 - S2). They exhibit coherent transient motion to the west (Figure 2), consistent

92 with SSE-induced deformation in this region ${ }^{4-12}$. The onset of the transient signature appears to be

93 delayed to the northwest, consistent with the along-strike SSE propagation imaged by inverting daily

94 static GPS data during the same period ${ }^{9}$ as well as the propagation of tremors to the northwest

95 (Figure 1), although it is not easy to visually identify a precise timing of the onset at each site. A

96 similar transient signature is not as clearly discernible in the north component of the position time

97 series as in the eastern component (Figure S1). However, guided by the eastern component as well as

98 the daily coordinates, we can discern the SSE-induced subtle transient deformation. A similar

99 coherent transient motion or permanent offset is not discernible at sites distant from the ETS area we

100 focus on (Figure S2), although they exhibit plenty of fluctuation with an amplitude of $\sim 5 \mathrm{~mm}$.

101 Hence, we concluded that our sub-daily GPS positions successfully captured previously reported

102 transient motion associated with the March to April 2017 SSE with a finer sampling interval. 
104 Spatiotemporal slip inversion using subdaily data

105 We performed a spatiotemporal slip inversion of the SSE using our cleaned 30-minute kinematic 106 GPS and a Kalman filter-based inversion method with a time-invariant temporal smoothness 107 parameter ${ }^{37-38}$ (See Method). At first, we attempted to determine the spatial and temporal smoothness 108 parameters by maximising the $\log$-likelihood ${ }^{38}$ (Figure S4), but the obtained slip evolution was 109 extremely oscillatory for reasons we discuss later. Hence, after testing several models with different 110 spatial and/or temporal smoothness (Figure S5), we present a preferred model of spatiotemporal slip 111 propagation as a possible scenario (Figure 3a, 3c, 3e, 4 and 5 and Videos S1 and S2) (See Method). 112 The final moment magnitude for the entire period is $\mathrm{M}_{\mathrm{w}} 6.5$ (Figure 3a). The moment rate history of 113 the SSE has two peaks in the 30-minute data inversion, consistent with two peaks of daily tremor 114 counts (Figure 4).

115 We divided the inferred spatiotemporal slip evolution using the 30-minute data into four stages based 116 on the moment rate history (Figure 4). We determined the initiation and termination of Stage 1 and 117 Stage 4, respectively, based on daily tremor counts (Figure 4), because our data post-processing 118 assumes that no SSEs occur when no tremors are detected (See Method). In the first stage, our model 119 shows slow crack-like isotropic growth of the SSE at 30-50-km depths with little migration (Stage 1; 120 Figures $3 \mathrm{c}$ and 5a and Video S2). This depth range is typical of short-term SSE occurrence in this 121 region ${ }^{1,4-6}$. Next, the isotropic growth is followed by an along-strike pulse-like migration towards the 122 northwest within the depth range (Stage 2; Figures $3 \mathrm{c}$ and $5 \mathrm{~b}$ and Video S2). The along-strike 123 migration continues and then the slip area expands to shallower depths at $\sim 48.5 \mathrm{~N}$, where the down124 dip limit of the locked zone is also shallower. Eventually, the slip area reaches the down-dip limit of 125 the locked zone ${ }^{39}$ (Stage 3; Figures 3c and 5c and Video S2). Finally, the slip migrates again in the 126 along-strike direction and terminates with a blurred slip rate pattern (Stage 4; Figures 3c and 5d and 127 Video S2). Our SSE model exhibits the onset of isotropic growth slightly earlier than the onset of the 128 tremor sequence (Figures 3e, 4 and 5a). One possible cause for them is the time-invariant 129 smoothness parameter for temporal slip rate (See Method), as has been reported ${ }^{37}$. Indeed, our 130 kinematic GPS time series exhibits a more abrupt onset of the transient surface motion than the 131 predicted SSE-induced motion at some sites (e.g. ALBH; Figure 2b), suggesting that the actual slip 132 onset is more abrupt than that inferred in this study but is not well constrained.

133 The transition of unbounded-bounded source growth was conceptualised from observations of 134 regular earthquakes ${ }^{40}$. Gomberg et al. ${ }^{41}$ has proposed that this growth mode transition is also the case 135 for slow earthquakes and reconciles controversial mechanical scaling of regular and slow 136 earthquakes ${ }^{19,21,41-42}$. The key idea is that slip growth is bounded (one-dimensional) or unbounded 137 (two-dimensional) depending on whether its slip area (hence moment) is large enough to fill the 138 limited depth range ${ }^{25,40}$, but direct observational evidence of the slip clearly exhibiting the 139 unbounded-bounded growth transition is limited ${ }^{6}$. The inferred SSE in this study shows the multi140 stage evolution, comprising the SSE growth within the limited depth range with little migration (i.e., 141 unbounded growth in Stage 1; Figures 3c, 3e and 5a and Video S2) and the subsequent unilateral 
142 migration after fully filling the depth range (bounded growth in Stages 2-4; Figures 3c, 3e, and 5b-

$1435 \mathrm{~d}$ and Video S2), which results in an elongated cumulative slip pattern in the along-strike direction

144 (Figure 3a and Video S1). Bletery and Nocquet ${ }^{6}$ already reported the similar unbounded-bounded

145 growth transition of a different SSE from the daily GPS data in Cascadia. This study adds supportive

146 observational evidence of the unbounded-bounded transition of the SSE growth mode with the

147 clearer unbounded growth process. Moreover, the crack-like up-dip growth at Stage 3 coincides with

148 the shallowing of the locking depth. The shallower locking depth is likely equivalent to the shallower

149 up-dip boundary of the rheologically permitted SSE zone, so the up-dip growth of the slip area

150 highlights the SSE growth mechanics controlled by fault rheology ${ }^{25}$. Our slip model implies the

151 presence of common mechanical factors behind the rupture kinematics of regular and slow

152 earthquakes, which needs testing through future investigation using the subdaily GPS data with more

153 events.

\section{Relationship of the inferred SSE process and tremors}

156 The inferred spatiotemporal slip rate evolution is also roughly concordant with the tremor migration

157 history (Figure 3e and Video S2), but their spatial relationship is different among the four stages. The 158 tremors occur near the centre of growth during Stage 1, but their epicentres are in the periphery of 159 the high slip rate area at Stage 2. The tremors and the high slip rate area overlap again during Stage

1603 , but the tremors cluster away from the main slip area in Stage 4. This feature implies multiple 161 tremor excitation mechanisms during one short-term SSE, such as direct loading by slow-slip and 162 stress increase ahead of the slip front ${ }^{8,12}$.

163 We compared the moment rate history with the daily tremor counts; both of them have consistent 164 time evolution at this resolution (Figure 4). Based on the sub-daily variation of tremor activity 165 (Figures $3 \mathrm{e}$ ), the presence of the sub-daily variation of slow slip rates has been proposed ${ }^{28}$. However, 166 our preferred slip rate model and the associated moment rate does not have such features (Figures $3 \mathrm{e}$, 1674 and 5). The test model in which more vigorous slip rate change is allowed demonstrates the 168 extremely oscillating moment rate history (Test 4 in Figure S5e), which compelled us to impose a 169 stronger temporal smoothing of slip rate during the entire period. However, as we further discuss in 170 the next section, this result does not exclude the previously proposed possibility of subdaily variation 171 of slip rates ${ }^{15,20-21,23,28-29}$.

\section{Comparison with daily data inversion and technical limitations of subdaily data}

174 For a comparison purpose, we performed the inversion using the daily coordinates and the same 175 method (Figures 3b, 3d and 3f and Videos S3 and S4) (See Method). Maximising the log-likelihood 176 yield a physically plausible spatiotemporal slip distribution in the daily data inversion. The cumulative slip patterns of the 30-min inversions (Figure 3a) are consistent with models for the same event derived from the daily static coordinate data in this study (Figure 3b) and Michel et al. ${ }^{9}$, demonstrating that the noisy subdaily coordinates have the ability to reproduce realistic slip patterns. 
180 The final moment magnitude for the entire period from the 30-min data inversion $\left(\mathrm{M}_{\mathrm{w}} 6.5\right.$, Figure

$1813 \mathrm{a})$ is slightly smaller than the estimate from the daily coordinates $\left(\mathrm{M}_{\mathrm{w}} 6.7\right.$; Figure $\left.3 \mathrm{~b}\right)$, but this 182 difference might be insignificant, given some uncertainties from the inversion parameter tuning.

183 Although a moment rate history with one peak was inferred in a daily inversion model in the 184 previous study ${ }^{9}$, our daily static and 30-minute data inversions produced the moment rate history 185 with two peaks (Figure 4), so the increase of the number of the moment rate peaks originates mainly 186 from the inversion strategy used in this study; the 30-minute kinematic coordinates themselves do 187 not have significant contribution to this improvement in temporal resolution. Nevertheless, in other 188 words, our 30-minute and daily data inversions demonstrate that the temporal resolution of the slip 189 and moment rates derived from the 30-minute coordinates can reach the comparable level to those 190 derived from the daily coordinates, which is astonishing given the generally-believed higher noise 191 level of kinematic GPS coordinates than that of daily coordinates.

192 We have pointed out the issue in the temporal smoothing scheme of the subdaily inversion so far, 193 which is, to a certain extent, responsible for the comparable time resolution of subdaily and daily slip 194 and moment rates. Yet, apart from this inversion technique issue, there exists a more important and 195 difficult issue; it is comprehensive understanding of non-tectonic signatures contaminating the 196 kinematic GPS positions such as tropospheric and ionospheric effects and multipath as well as other 197 enigmatic site-specific effects ${ }^{30}$. Although we largely mitigated these effects during the positioning 198 and its post-processing, the failure to determine some hyperparameters in the slip inversion strongly 199 suggests the presence of significant unmodelled non-tectonic signatures left in the kinematic data; 200 such non-tectonic noises were formally described by the spatiotemporally rough slip distribution by 201 exploiting the non-parametric formulation of slip and slip rate ${ }^{37-38}$. From the daily data, the plausible 202 slip evolution was inferred with the hypermeters determined by maximising the log-likelihood, 203 which also highlights the presence of unmodelled noise in the "cleaned" 30-minute kinematic 204 coordinates. This study has not fully addressed this issue and further improvements in modelling 205 and/or removing such noise are crucial to extract subdaily variations of slip rate and to detect 206 isolated events lasting shorter than one day. At this point, this study provides the minimum recipe of 207 the non-tectonic noise mitigation as a milestone for such future investigation. 
209 Methods

210 GPS kinematic analysis

211 We determined the relative position of the GPS sites with respect to the reference site DRAO (Fig.

212 S3) every 30 seconds between 11 March (day of the year (DOY) 070) and 17 April (DOY 107) 2017

213 using the TRACK program of GAMIT/GLOBK ${ }^{43-45}$ and the IGS final satellite orbit. We only

214 employed GPS observables as those from other satellite systems are not available at some sites. We

215 used observables from GPS satellites with an elevation angle of 15 degrees or above. We used the

216 Vienna Mapping Function ${ }^{46}$, the IGS ionosphere products (i.e. IONEX) and FES2004 tidal model ${ }^{47-48}$

217 in the centre of mass of the solid Earth to account for tropospheric, ionospheric, and tidal effects,

218 respectively. We took a priori position of each site on each day from the static daily solution of the

219 Nevada Geodetic Laboratory (NGL) ${ }^{49}$ or, if not available, the header of each RINEX file with its

220 uncertainty assumed to be $10 \mathrm{~cm}$ or $50 \mathrm{~cm}$, respectively. The random-walk parameter of the position

221 determination was set to $1 \mathrm{~cm} / \sqrt{30 \text { seconds }}$. First, we divided the 64 kinematic GPS sites into

222 eight groups and performed kinematic analysis for each group separately relative to the reference site

223 in each group (Figure S3). Then, we performed another kinematic analysis for eight sites, consisting

224 of a reference site for each group at the first step, with respect to DRAO. Finally, we took the

225 summation of the solutions of the two steps in the geocentric coordinate system to obtain the

226 kinematic position relative to DRAO. We take this two-step approach to exploit the advantage of the

227 relative positioning method, which mitigates the effects of the atmosphere on the microwave

228 pathway more efficiently for a shorter baseline. The obtained relative position (black time series in

229 Figure S6a) is mostly free from the rigid plate motion of the North American plate.

\section{Data post-processing}

232 We performed several steps of post-processing of the kinematic GPS coordinates to mitigate non233 tectonic noise due to recognised sources in previous studies and some random outliers (Figures S6 234 and S7). First, we applied a sidereal filtering technique to mitigate coordinate fluctuations caused by 235 multipath $^{33-35}$. We assumed the repeat period of the sidereal filter to be 86154 seconds $^{35}$ and

236 generated a sidereal filter by stacking six 86154-second length time series made of those during 237 DOY 070-072 and 104-107. No tremors were detected during these periods by the PNSN network, 238 so we assumed no SSE (accordingly no SSE-induced site motion) during these periods, which is an 239 important assumption for making sidereal filters ${ }^{33-35}$. This assumption might be incorrect because an 240 SSE without tremors has been reported before ${ }^{7}$. However, as presented in Figure S6a, the fluctuation 241 of our sub-daily coordinates was greatly reduced by sidereal filtering, so we accepted this 242 assumption in this study. To obtain the 86154-second length series and achieve a shift of time series 243 by multiple of this length using the 30 -second coordinates, we interpolated the 30 -second interval 244 series linearly. After stacking the six series, we concatenated the stacked series until it became longer 245 than the period of interest (i.e. DOY 073-103). Then, we demeaned, detrended and decimated it to a 24630 -second interval. Finally, we subtracted the constructed sidereal filter from the position time series 
247 during the period of interest (red time series in Figure S6a).

248 Then, we removed the common-mode error (CME), mainly due to instability of the reference site 249 and the coordinate system ${ }^{36}$, using an independent component analysis (ICA)-based approach ${ }^{50}$. We

250 let all three components share the same mode. We determined the number of independent components (ICs) to be 25 after trial and errors. We found that we could not extract some modes representing CME with too few ICs, while some modes for CME were decomposed into spatially non-uniform patterns by gradually increasing the number of ICs. This competition provided us with a plausible number of ICs. Eventually, we identified three modes corresponding to CME (Figure S7), which were subsequently removed from the sidereal-filtered data (blue time series in Figure S6a). Then, we applied the same ICA-based CME removal for the eight subsets of the kinematic sites used in the kinematic positioning analysis. For this step, the number of ICs was set to the number of data points for simplicity, and we removed one or zero modes by inspecting the spatial pattern of the decomposed modes (light blue time series in Figure S6a). We decided to include this step as we happened to find such a signature during the whole network CME removal, although the origin of these intra-group common-mode errors is elusive. We applied the post-processing explained so far to the relative position in the geocentric coordinate system to avoid undesirable effects caused by applying the CME removal to positions expressed in the site-to-site variable topocentric coordinate system (i.e. the intuitive North-East-Vertical system). We did not remove any other modes, even if their spatial pattern did not look significant, because ICA could force to decompose the temporally variable deformation pattern due to the spatially migrating SSE of interest into multiple timeinvariant spatial patterns. After CME removal, we converted the coordinate system of the position to the local topocentric coordinate system (light blue time series in Figure S6b). Next, we removed the diurnal variation of the position time series expressed in the local topocentric coordinate system using a loess-based seasonal-trend decomposition method called STL ${ }^{51-52}$ (black time series in Figure S6b). Here, the diurnal variation was estimated as the seasonal component, and we used the estimated trend and residual components for subsequent analysis to avoid dropping any short-term transient left in the residual component. Then, we decimated the 30-second interval position time series into a 30-minute interval (blue and pink time series in Figure S6b) after applying the low-pass filter with a cut-off period of $1 \mathrm{~h}$ (yellow time series in Figure S6b). This decimation was required to reduce computational burden for the subsequent slip inversion. As the time series to be filtered contain the permanent offset due mainly to the SSE, we applied a Hanning window with a width of one day at both ends of the time series (i.e. DOYs 073 and 103) prior to low-pass filtering using Seismic Analysis Code (SAC) ${ }^{53-54}$. After the low-pass filter was completed, we omitted the epochs used for tapering. We confirmed that no tremors were detected during these two days. In this decimation step, we also omitted epochs purely predicted by the Kalman filter - smoother (i.e. epochs at which no raw observables were available) during the kinematic positioning analysis. Even after these data cleaning processes, we identified spurious outliers in the position time series, 
so we removed some of them satisfying the following criterion:

$$
\left|u_{i}-\frac{q_{1}+q_{3}}{2}\right|>n * \frac{q_{3}-q_{1}}{2}
$$

where $u_{i}$ is the displacement at the $i$ th epoch, $q_{1}$ and $q_{3}$ are the 25 and 75 percentile values of the position time series, respectively, and $n$ is a threshold controlling how strict or loose we impose the outlier criterion (blue dots in Figure S6b). We tested some different values for the threshold and finally preferred $n=5$ to avoid removing the transient pattern likely representing the SSE-induced site motion, which means we removed epochs that deviated significantly from the main trend. We kept epochs where both the east and north components did not satisfy this criterion (pink time series in Figure S6b).

\section{Spatiotemporal slip inversion}

We employed a Kalman-filter-based inversion scheme which allows non-parametric inference of temporal slip evolution ${ }^{38}$. The forward model we used is described in Fukuda et a ${ }^{37}$ and Fukuda ${ }^{55}$, except for a hyperparameter, $\alpha^{2}$, which controls the temporal smoothness of slip and slip rate; in this study we set $\alpha^{2}$ to be time-invariant. We inverted the two horizontal components of the cleaned position time series and did not use the vertical component because there is a significant trade-off between the vertical position and the zenith tropospheric delay estimates during the kinematic GPS analysis. We modelled the Juan de Fuca slab interface down to $60-\mathrm{km}$ depths using the slab 2.0 mode $^{56}$. The slab surface was tessellated by triangular subfaults (Figure S8), and we computed the elastic deformation in response to their unit slip in the relative convergence direction of the converging plates (Figure 1) ${ }^{57}$ using a homogeneous elastic half-space model ${ }^{58}$. For simplicity, we ignored the elastic heterogeneity ${ }^{59-60}$. To alleviate the non-uniqueness of the spatial slip pattern and stabilise the inversion, we imposed a spatial smoothness constraint on the slip distribution. In this study, we took a singular value decomposition approach of Green's functions to create a set of smooth basis functions ${ }^{37,61}$ and determined their amplitudes in the inversion. In this approach, the spatial smoothness of the estimated slip is controlled by the number of basis functions, $M$, and we can separately control the spatial and temporal smoothness. The temporal smoothness is controlled by one time-invariant hyperparameter $\alpha^{2}$ controlling the range of slip rate change from one epoch to the next ${ }^{37-38,55}$. With larger $M$ and $\alpha^{2}$, the resultant spatial and temporal slip distributions become rougher, respectively (Figure S5). We simultaneously modelled the site-specific random-walk motion of the benchmark ${ }^{38}$ and translation of the entire network, which were controlled by one scale factor for each ${ }^{37,55}$. Although we assumed that a substantial portion of the CME had already been removed, we included the latter term in the slip inversion as well because our CME removal by the postprocessing was based on the "estimation" of ICA, which potentially missed some CME. Typically, these four hyperparameters, namely, $M, \alpha^{2}$, and scale factors for the random-walk and the translation components, needs optimising by maximising the likelihood ${ }^{37-38,55}$. However, in this study, we assumed $1 \mathrm{~mm} / \sqrt{y r}$ and $5 \mathrm{~mm}$ for the scale factors of random-walk ${ }^{55}$ and the translation 
322 components, respectively. We first attempted to determine $M$ and $\alpha^{2}$ by maximising the likelihood,

323 but this resulted in an extremely rough unphysical spatiotemporal slip distribution (Figures S4 and 324 S5). Hence, we determined the preferred combination of $M$ and $\alpha^{2}$ to be 18 and $1000 \mathrm{~m}^{2} / \mathrm{year}^{3}$, 325 respectively, by inspecting an inflexion point of $\alpha^{2}$ versus $\log$-likelihood curves with various $M$ 326 (Figure S4). Here, we assumed that a gentler increase in log-likelihood beyond the preferred $M$ and $327 \quad \alpha^{2}$ reflected data fitting improvement by describing the unmodelled enigmatic signature in the data.

328 We present four test models with different $M$ and $\alpha^{2}$ values, which demonstrate rougher or smoother 329 spatiotemporal slip patterns in Figure S5. The failure of maximum-likelihood determination of these 330 hyperparameters indicates that the formal covariance matrix does not reasonably estimate the 331 uncertainty for slip and slip rates. Hence, we preferred not to provide formal estimation errors for our 332 results and exclusively discussed the obvious features.

333 We applied the same inversion scheme to the daily GPS coordinates provided by NGL ${ }^{49}$. We 334 converted the daily coordinates to those relative to DRAO to realise the same reference frame as the 335 kinematic coordinates (Figures 3b, 3d, 3f and 4 and Videos S3 and S4). We used the hyperparameters 336 determined by maximising the log-likelihood because it yielded the physically plausible 337 spatiotemporal slip evolution. The same number of the basis functions, $M=18$, as the preferred 338 model of the subdaily inversion yielded the maximum log-likelihood in the daily inversion. The 339 temporal smoothness parameter is estimated to be $\alpha^{2}=1778.28 \mathrm{~m}^{2} / \mathrm{year}^{3}$. The inferred model 340 reasonably fits the daily GPS coordinates (Figures S9 - S11).

\section{References}

349 1. Gomberg, J., the Cascadia 2007 and Beyond Working Group, Slow-slip phenomena in $350 \quad$ Cascadia from 2007 and beyond: A review. GSA Bull. 122, 963-978 (2010).

351 2. Obara, K., Kato, A. Connecting slow earthquakes to huge earthquakes. Science 353, 253$257(2016)$.

353 3. Bürgmann, R. The geophysics, geology and mechanics of slow fault slip. Earth Planet. Sci. $354 \quad$ Lett. 495, 112-134 (2018).

355 4. Dragert, H. , Wang, K., James, T. S. A silent slip event on the deeper Cascadia subduction 356 interface. Science 292, 1525-1528 (2001).

357 5. Wech, A. G., Creager, K. C., Melbourne, T. I. Seismic and geodetic constraints on Cascadia $358 \quad$ slow slip. J. Geophys. Res. Solid Earth 114, B10316 (2009).

359 6. Bletery, Q., Nocquet, J. M. Slip bursts during coalescence of slow slip events in Cascadia. 
Nat. Commun. 11, 2159; 10.1038/s41467-020-15494-4 (2020).

7. Wech, A. G., Bartlow, N. M. Slip rate and tremor genesis in Cascadia. Geophys. Res. Lett. 41, 392-398 (2014).

8. Bartlow, N. M., Miyazaki, S., Bradley, A. M., Segall, P. Space-time correlation of slip and tremor during the 2009 Cascadia slow slip event. Geophys. Res. Lett. 38, L18309 (2011). Michel, S., Gualandi, A., Avouac, J. P. Interseismic coupling and slow slip events on the Cascadia megathrust. Pure Appl. Geophys. 176, 3867-3891 (2019).

10. Schmidt, D. A., Gao, H. Source parameters and time-dependent slip distributions of slowslip events on the Cascadia subduction zone from 1998 to 2008. J. Geophys. Res. Solid Earth 115, B00A18 (2010).

11. Hall, K., Houston, H., Schmidt, D. Spatial comparisons of tremor and slow slip as a constraint on fault strength in the northern Cascadia subduction zone. Geochem. Geophys. Geosys. 19, 2706-2718 (2018).

12. Hall, K., Schmidt, D., Houston, H. Peak tremor rates lead peak slip rates during propagation of two large slow earthquakes in Cascadia. Geochem. Geophys. Geosys. 20, 4665-4675 (2019).

13. Nishimura, T., Matsuzawa, T., Obara, K. Detection of short-term slow slip events along the Nankai Trough, southwest Japan, using GNSS data. J. Geophys. Res. Solid Earth 118, 31123125 (2013).

14. Rousset, B., Bürgmann, R., Campillo, M. Slow slip events in the roots of the San Andreas fault. Sci. Adv. 5, eaav3274 (2019).

15. Rousset, B., Fu, Y., Bartlow, N., Bürgmann, R. Week-Long and Year-Long Slow Slip and Tectonic Tremor Episodes on the South Central Alaska Megathrust. J. Geophys. Res. Solid Earth 124, 13392- 13403 (2019)

16. Rogers, G., Dragert, H. Episodic tremor and slip on the Cascadia subduction zone: The chatter of silent slip. Science 300, 1942-1943 (2003).

17. Ghosh, A. Huesca-Pérez, E., Brodsky, E., Ito, Y. Very low frequency earthquakes in Cascadia migrate with tremor. Geophys. Res. Lett. 42, 3228-3232 (2015).

18. Shelly, D., Beroza, G., Ide, S. Non-volcanic tremor and low-frequency earthquake swarms. Nature 446, 305-307 (2007).

19. Michel, S., Gualandi, A., Avouac, J. P. Similar scaling laws for earthquakes and Cascadia slow-slip events. Nature 574, 522-526 (2019).

20. Frank, W. B. Slow slip hidden in the noise: The intermittence of tectonic release. Geophys. Res. Lett. 43, 10125-10133 (2016)

21. Frank, W. B., Brodsky, E. E. Daily measurement of slow slip from low-frequency earthquakes is consistent with ordinary earthquake scaling. Sci. Adv. 5, eaaw9386 (2019)

22. Nishikawa, T. et al. The slow earthquake spectrum in the Japan Trench illuminated by the Snet seafloor observatories. Science, 365, 808-813 (2019). 
23. Fujita, M., Nishimura, T., Miyazaki, S. Detection of small crustal deformation caused by slow slip events in southwest Japan using GNSS and tremor data. Earth Planets Space 71, 96; 10.1186/s40623-019-1075-x (2019).

24. Rousset, B., Bürgmann, R., Campillo, M. Slow slip events in the roots of the San Andreas fault. Sci. Adv. 5, eaav3274 (2019).

25. Gao, X., Wang, K. Rheological separation of the megathrust seismogenic zone and episodic tremor and slip. Nature 543, 416-419 (2017).

26. Liu, Y., Rice, J. R. Aseismic slip transients emerge spontaneously in three-dimensional rate and state modeling of subduction earthquake sequences. J. Geophys. Res. Solid Earth 110, B08307 (2005)

27. Segall, P., Rubin, A. M., Bradley, A. M., Rice, J. R. Dilatant strengthening as a mechanism for slow slip events. J. Geophys. Res. Solid Earth 115, B12305 (2010)

28. Jolivet, R., Frank, W. B. The transient and intermittent nature of slow slip. $A G U A d v$. 1, e2019AV000126 (2020).

29. Hawthorne, J. C., Rubin, A. M. Short-time scale correlation between slow slip and tremor in Cascadia. J. Geophys. Res. Solid Earth 118, 1316-1329 (2013).

30. Bock, Y., Nikolaidis, R. M., de Jonge, P. J., Bevis, M. Instantaneous geodetic positioning at medium distances with the Global Positioning System. J. Geophys. Res. Solid Earth 105, 28223-28253 (2000).

31. Twardzik, C., Vergnolle, M., Sladen, A., Avallone, A. Unravelling the contribution of early postseismic deformation using sub-daily GNSS positioning. Sci. Rep. 9, 1775; 10.1038/s41598-019-39038-z (2019).

32. Jiang, J., Bock, Y., Klein, E. Coevolving early afterslip and aftershock signatures of a San Andreas fault rupture. Sci. Adv. 7, eabc1606 (2021).

33. Choi, K., Bilich, A., Larson, K. M., Axelrad, P. Modified sidereal filtering: Implications for high-rate GPS positioning. Geophys. Res. Lett. 31, L22608 (2004).

34. Larson, K. M., Bilich, A., Axelrad, P. Improving the precision of high-rate GPS. J. Geophys. Res. Solid Earth 112, B05422 (2007).

35. Ragheb, A. E., Clarke, P. J., Edwards, S. J. GPS sidereal filtering: coordinate- and carrierphase-level strategies. J. Geodesy 81, 325-335 (2007).

36. Wdowinski, S., Bock, Y., Zhang, J., Fang, P., Genrich, J. Southern California permanent GPS geodetic array: Spatial filtering of daily positions for estimating coseismic and postseismic displacements induced by the 1992 Landers earthquake. J. Geophys. Res. Solid Earth 102, 18057-18070 (1997).

37. Fukuda, J., Miyazaki, S., Higuchi, T., Kato, T. Geodetic inversion for space--time distribution of fault slip with time-varying smoothing regularization. Geophys. J. Int. 173, 25-48 (2008). 
435

38. Segall, P., Matthews, M. Time dependent inversion of geodetic data. J. Geophys. Res. Solid Earth 102, 22391-22409 (1997).

39. Li, S., Wang, K., Wang, Y., Jiang, Y., Dosso, S. E. Geodetically inferred locking state of the Cascadia megathrust based on a viscoelastic Earth model. J. Geophys. Res. Solid Earth 123, 8056-8072 (2018).

40. Scholz, C. H. Scaling laws for large earthquakes: Consequences for physical models. Bull. Seismol. Soc. Am. 72, 1-14 (1982).

41. Gomberg, J., Wech, A., Creager, K., Obara, K., Agnew, D. Reconsidering earthquake scaling. Geophys. Res. Lett. 43, 6243-6251 (2016).

42. Ide, S., Beroza, G., Shelly, D., Uchide, T. A scaling law for slow earthquakes. Nature 447, 76-79 (2007).

43. Herring, T. A., King, R. W., Floyd, M. A., McClusky, S. C. Introduction to GAMIT/GLOBK Release 10.7. http://geoweb.mit.edu/gg/Intro_GG.pdf. (2018).

44. Herring, T. A., King, R. W., Floyd, M. A., McClusky, S. C. GAMIT Reference Manual GPS Analysis at MIT Release 10.7. http://geoweb.mit.edu/gg/GAMIT_Ref.pdf. (2018).

45. T. A. Herring, M. A. Floyd, R. W. King, S. C. McClusky, GLOBK Reference Manual Global Kalman filter VLBI and GPS analysis program Release 10.6. http://geoweb.mit.edu/gg/GLOBK_Ref.pdf. (2015).

46. Boehm, J., Werl, B., Schuh, H. Troposphere mapping functions for GPS and very long baseline interferometry from European Centre for Medium-Range Weather Forecasts operational analysis data. J. Geophys. Res. Solid Earth 111, B02406 (2006).

47. Letellier, T. Etude des ondes de marée sur les plateaux continentaux. Dissertation, Université de Toulouse III (2004).

48. Lyard, F., Lefevre, F., Letellier, T., Francis, O. Modelling the global ocean tides: modern insights from FES2004. Ocean Dyn. 56, 394-415 (2006).

49. Blewitt, G., Hammond, W. C., Kreemer, C. Harnessing the GPS data explosion for interdisciplinary science. Eos, 99; 10.1029/2018EO104623 (2018).

50. Hyvärinen, A., Oja, E. Independent component analysis: algorithms and applications. Neural Netw. 13, 411-430 (2000).

51. Pedregosa, F. et al. Scikit-learn: Machine Learning in Python, JMLR 12, 2825-2830 (2011).

52. Cleveland, R. B., Cleveland, W. S., McRae, J. E., Terpenning, I. STL: A seasonal-trend decomposition procedure based on loess. J. Official Stat. 6, 3-73 (1990).

53. Goldstein, P., Snoke, A. SAC Availability for the IRIS Community. Incorporated Research Institutions for Seismology Data Management Center Electronic Newsletter. https://ds.iris.edu/ds/newsletter/vol7/no1/193/sac-availability-for-the-iris-community/. (2005).

54. Helffrich, G., Wookey, J., Bastow, I. The Seismic Analysis Code. (Cambridge University Press, 2013). 
55. Fukuda, J. Variability of the space-time evolution of slow-slip events off the Boso Peninsula, Central Japan, from 1996 to 2014. J. Geophys. Res. Solid Earth 123, 732-760 (2018).

56. Hayes, G. Slab2 - A Comprehensive Subduction Zone Geometry Model. U.S. Geological Survey data release https://doi.org/10.5066/F7PV6JNV (2018).

57. DeMets, C., Gordon, R. G., Argus, D. F. Geologically current plate motions. Geophys. J. Int. 181, 1-80 (2010).

58. Thomas, A. L. Poly3D: A three-dimensional, polygonal element, displacement discontinuity boundary element computer program with applications to fractures, faults, and cavities in the Earth's crust. MS thesis, Stanford Univ. (1993).

59. Williams, C. A. Wallace, L. M. The impact of realistic elastic properties on inversions of shallow subduction interface slow-slip events using seafloor geodetic data. Geophys. Res. Lett. 45, 7462-7470 (2018).

60. Bartlow, N. M. A long-term view of episodic tremor and slip in Cascadia. Geophys. Res. Lett. 47, e2019GL085303 (2020).

61. Segall, P., Bürrgmann, R., Matthews, M. Time dependent triggered afterslip following the 1989 Loma Prieta earthquake. J. Geophys. Res. Solid Earth 105, 5615-5634 (2000).

62. Wessel, P., Smith, W. H. F., Scharroo, R., Luis, J., Wobbe, F. Generic mapping tools: improved version released. EOS Trans. Am. Geophys. Union 94, 409-410. (2013).

\section{Acknowledgements}

We acknowledge T. Herring and S. Shimada for their technical assistance in using TRACK and S. Li for providing his research outcome ${ }^{39}$. We used Generic Mapping Tools ${ }^{62}$ to draft the figures. This study is supported by the Japan Society of the Promotion of Science (JSPS) Grants-in-Aid for Scientific Research (KAKENHI) JP21K14007 (Y.I.) and the Director Discretionary Fund of Earthquake Research Institute, the University of Tokyo (Fiscal Year 2020) (Y.I., Y.A., J.F.). We used the computer systems of the Earthquake and Volcano Information Center of the Earthquake Research Institute, the University of Tokyo.

\section{Author contributions}

Y.I. designed the study, performed all the data analysis and modelling, modified the slip inversion code, interpreted and discussed the results, and drafted the manuscript. Y.A. helped design the study and draft the manuscript and interpreted and discussed the results. J.F. prepared the original slip inversion code, interpreted and discussed the results, and helped draft the manuscript.

\section{Competing interests}

The authors declare no competing interests. 


\section{Additional Information}

\section{Data availability}

513 The RINEX files were retrieved via UNAVCO (https://data.unavco.org/archive/gnss/rinex/obs/) and

514 the Western Canada Deformation Array (WCDA; ftp://wcda.pgc.nrcan.gc.ca) servers. FES2004

515 ocean tide model is available at http://holt.oso.chalmers.se/loading/. TRACK in GAMIT/GLOBK

$516 \operatorname{program}^{43-45}$ and Seismic Analysis Code (SAC) $)^{53-54}$ are available upon request to the developers at

517 http://geoweb.mit.edu/gg/license.php and http://ds.iris.edu/ds/nodes/dmc/forms/sac/, respectively. We

518 made our slip and slip rate files available at $\operatorname{xxxxx~(ready~at~the~time~of~publication).~}$

519

520 Supplementary Information

521 Figures S1 - S11 and Videos S1 - S4

522

523 


\section{$524 \quad$ Figure and figure legends}

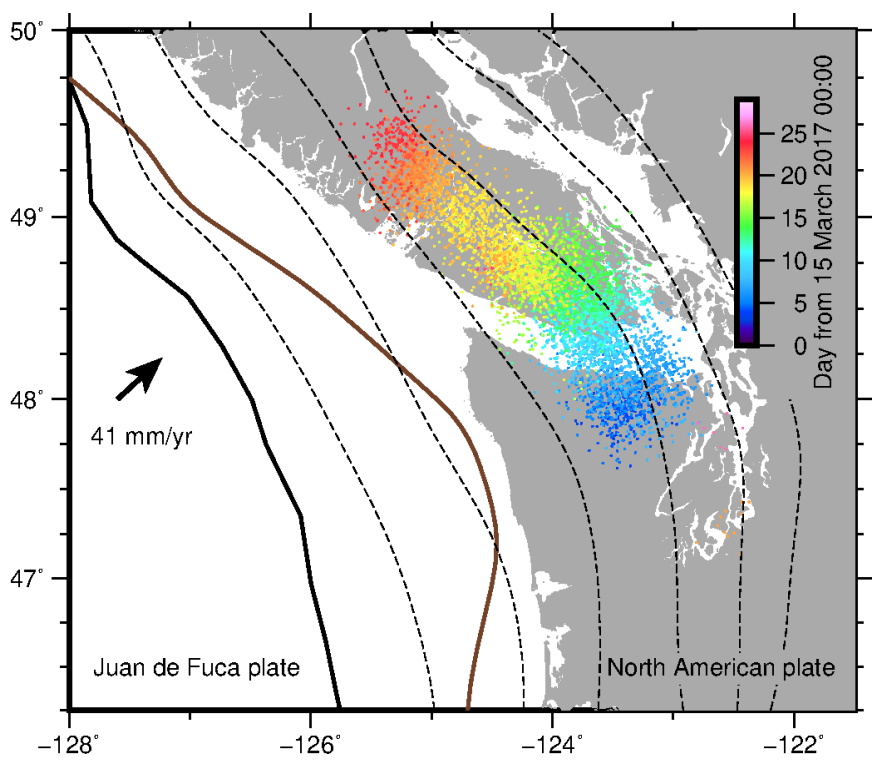

528 Figure 1. Tectonic setting of Cascadia subduction zone. The solid bold black line indicates the trench of the two converging plates as labelled. The broken lines indicate slab surface depth contours (10 $530 \mathrm{~km}$ interval from $10-\mathrm{km}$ depth $)^{56}$. The solid brown line outlines the interseismic locking depth ${ }^{39}$, 531 defined as a contour of locking ratio $=0.7$. The small dots color-coded with time indicate tremor 532 epicentres detected by PNSN (https://pnsn.org/tremor). 

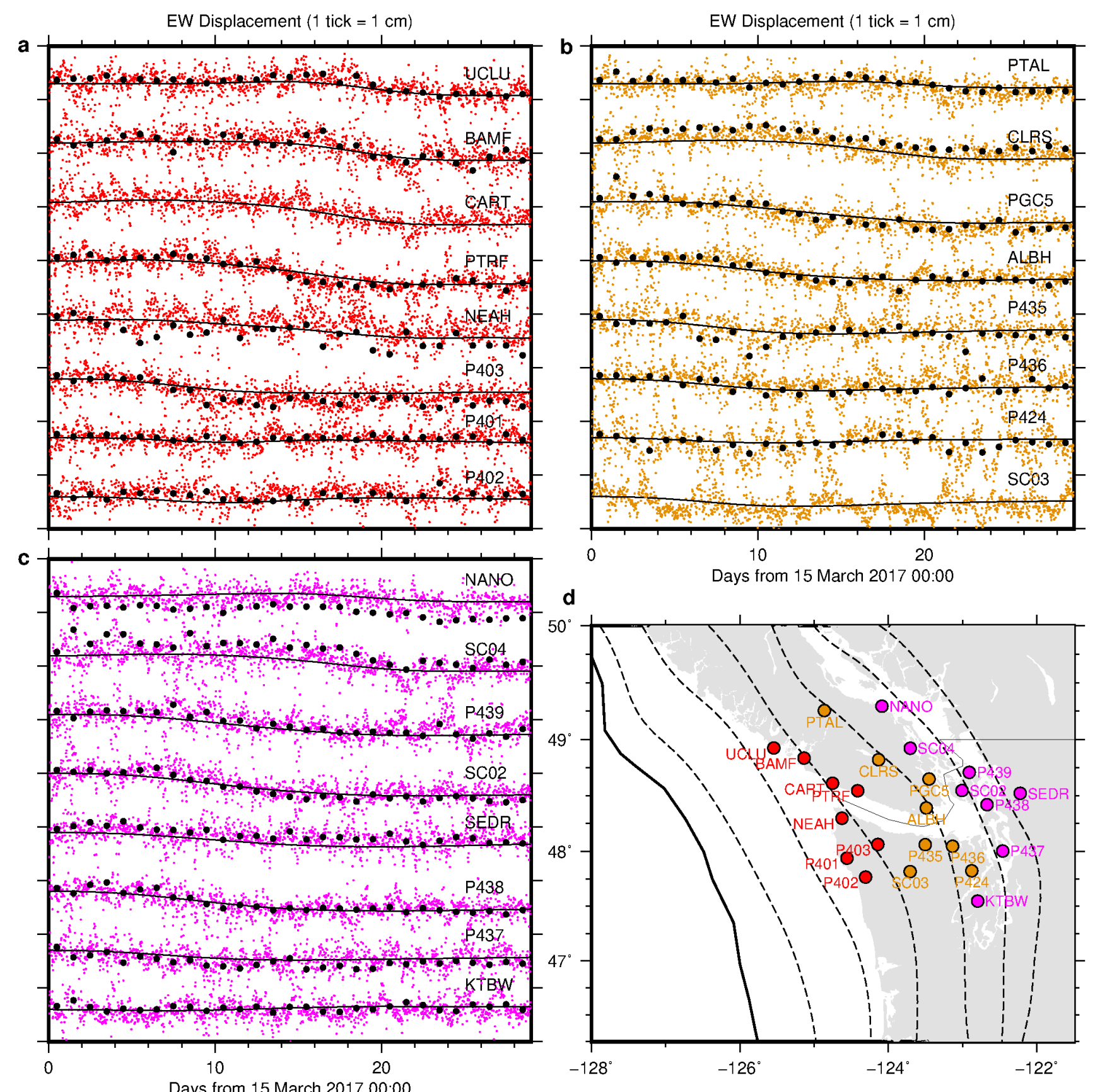

Days from 15 March 2017 00:00

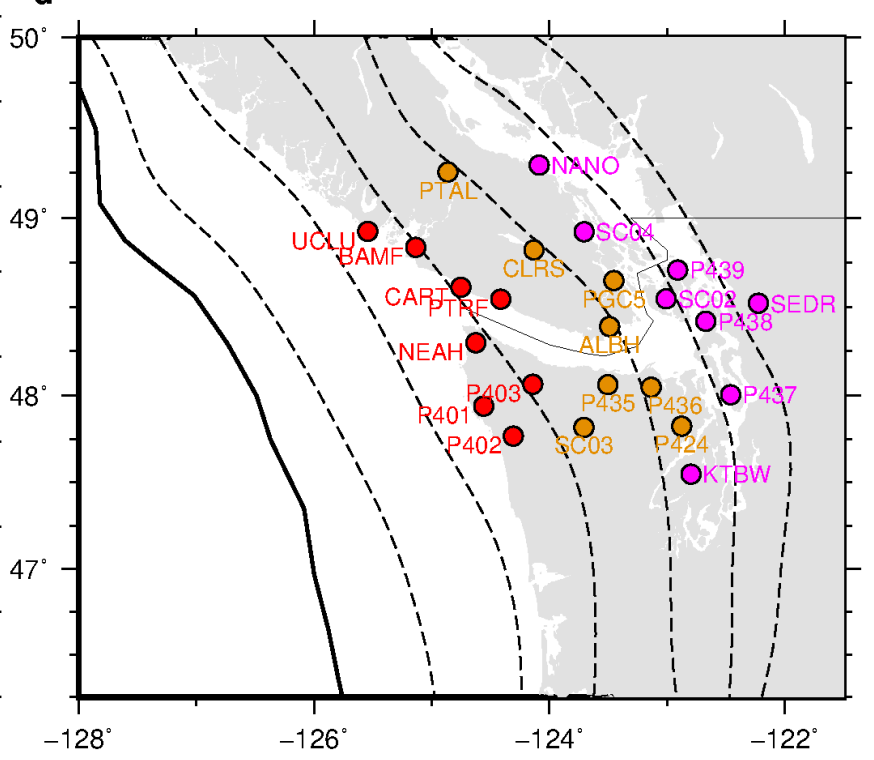

537 Figure 2. Kinematic GPS data at a 30-minute interval and fitting by spatiotemporal slip model. a - c.

538 The coloured dots indicate the post-processed east component of kinematic 30-min GPS positions,

539 further corrected for whole network translation estimated in 30-min slip inversion (see Method). The

540 overlying black dots indicate daily static east component which were corrected for whole network

541 translation estimated in the daily slip inversion (see Method). The daily solutions happen to be

542 unavailable at CART and SC03. The overlying black solid lines indicate the predicted motion due to

543 the fault slip. d. Site location. The coloured circles indicate the site location of the time series with

544 the same colour in $\mathbf{a}-\mathbf{c}$. 

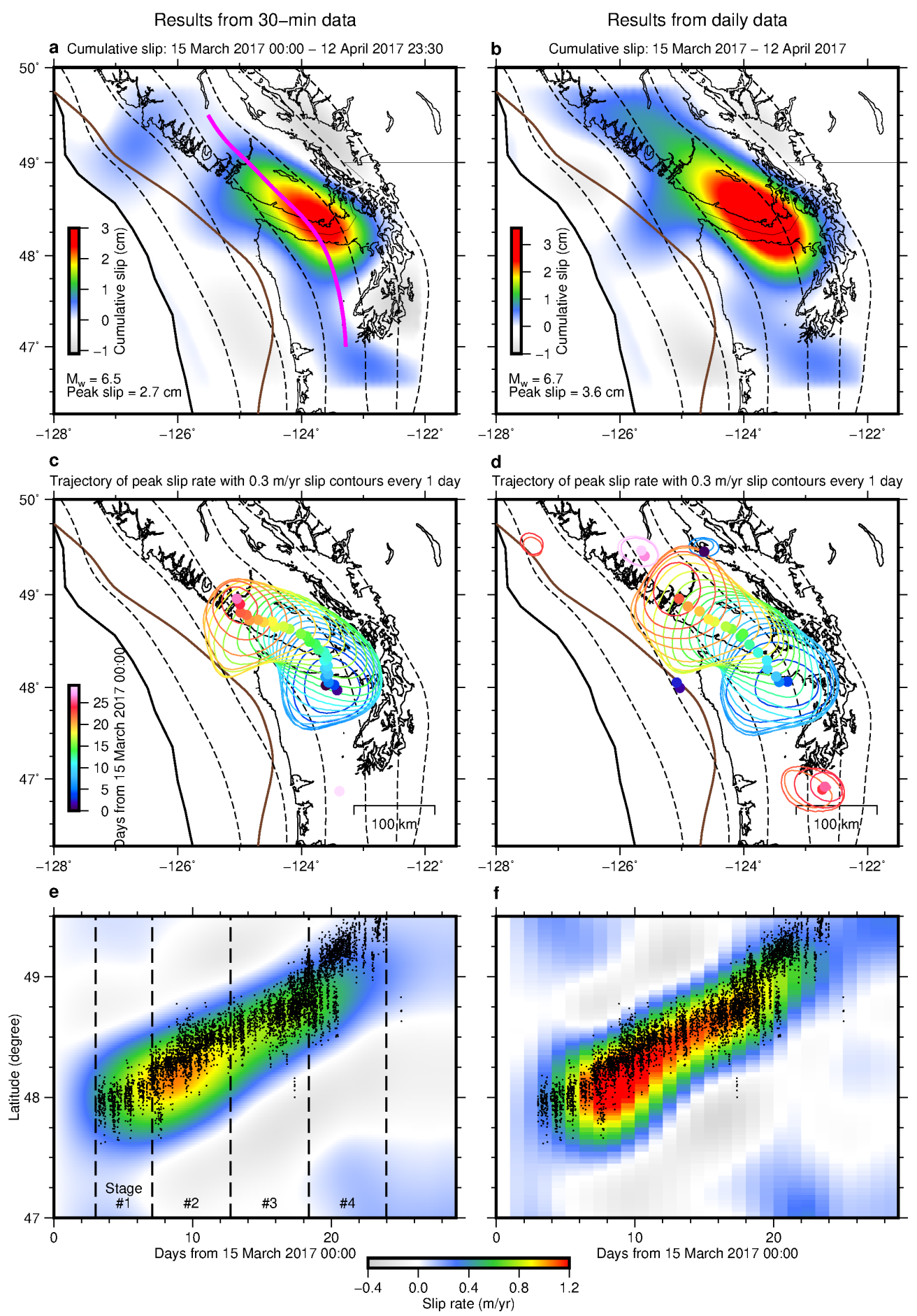

Figure 3. Comparison of inversion results using 30-min and daily data. Left (a, c, and e) and right (b, d, and f) columns indicate results from inversions of 30-min kinematic and daily static GPS

549 positions, respectively. a - b. Cumulative slip of the March to April 2017 SSE. The thick purple 550 curve in panel a indicates the location of the profile for Figures $3 \mathrm{e}-\mathrm{f}$. $\mathbf{c}-\mathbf{d}$. Collection of slip rate contours of $0.3 \mathrm{~m} / \mathrm{yr}$ every day is color-coded with time. Colour-coded dots indicate the trajectory of peak slip rate location. The colour of trajectory and contours indicates the time. $\mathbf{e}-\mathbf{f}$. Temporal evolution of slip rate along a profile along $35-\mathrm{km}$ slab interface depth (the purple curve in Figure $3 \mathrm{a}$ ) with tremors (dots), epicentres of which are located above the $30-$ to $40-\mathrm{km}$ depths slab interface. 


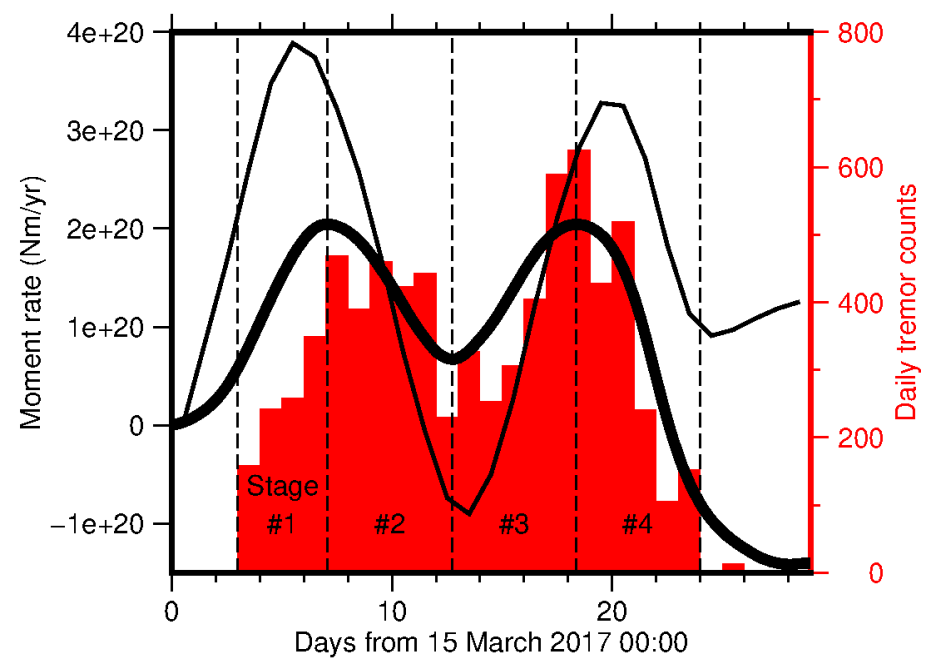

556 Figure 4. Moment rate history (solid curves) and daily tremor counts (red histogram). The thick and 557 thin solid curves indicate moment rate history of 30-min and daily data inversions, respectively. The 558 vertical broken lines indicate the boundaries of the four stages (Figures $5 \mathrm{a}-\mathrm{d}$ ). 

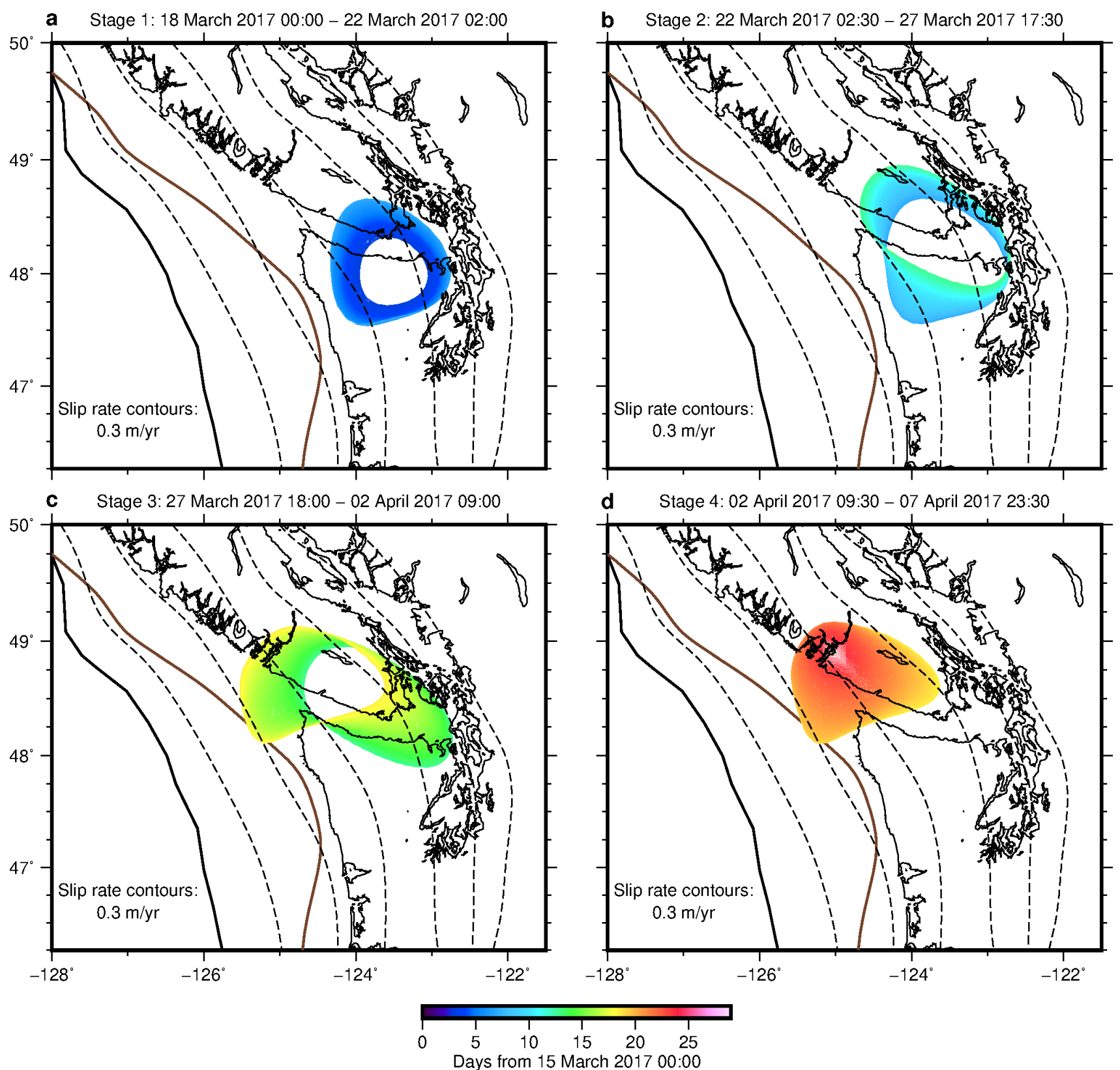

561 Figure 5. Spatiotemporal slip propagation drawn as collection of slip rate contours of the same slip 562 rate during four stages as labelled. Collection of slip rate contours of $0.3 \mathrm{~m} / \mathrm{yr}$ at a 30 -minute interval 563 is colour-coded with time. 


\title{
Supplementary Information
}

\section{Imaging evolution of Cascadia slow-slip event by high-rate GPS}

\author{
Yuji Itoh $^{1, *}$, Yosuke Aoki ${ }^{1}$, Junichi Fukuda ${ }^{1}$ \\ ${ }^{1}$ Earthquake Research Institute, the University of Tokyo, Japan \\ *Corresponding author: yitoh@eri.u-tokyo.ac.jp
}



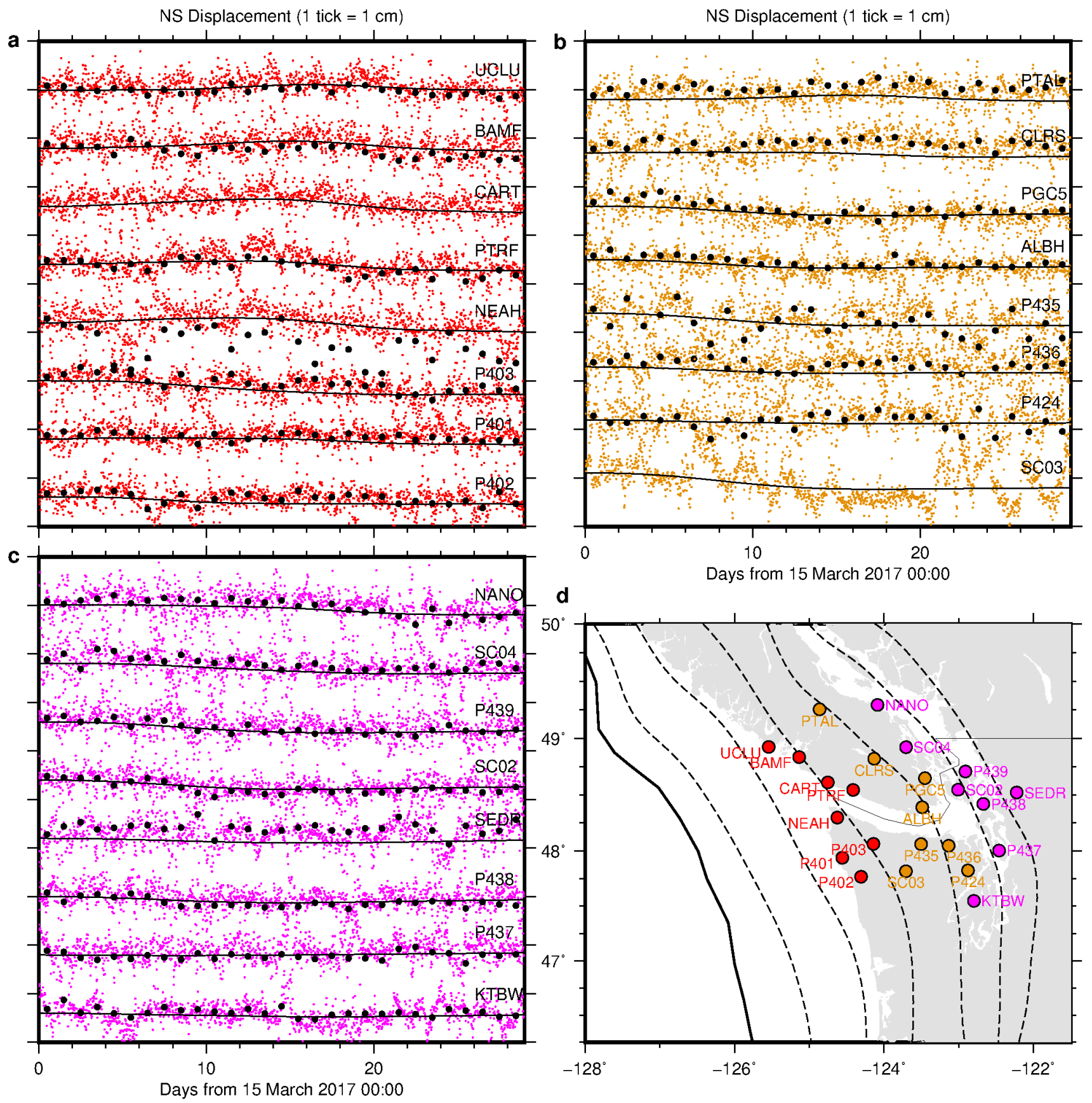

Figure. S1. Kinematic GPS data at a 30-minute interval and fitting by spatiotemporal slip model. ac. The coloured dots indicate the post-processed north component of kinematic 30-min GPS positions, further corrected for whole network translation estimated in 30-min slip inversion (see Method). The overlying black dots indicate daily static east component which were corrected for whole network translation estimated in the daily slip inversion (see Method). The daily solutions happen to be unavailable at CART and SC03. The overlying black solid lines indicate the predicted motion due to the fault slip. d. Site location. The coloured circles indicate the site location of the time series with the same colour in $\mathbf{a}-\mathbf{c}$. 

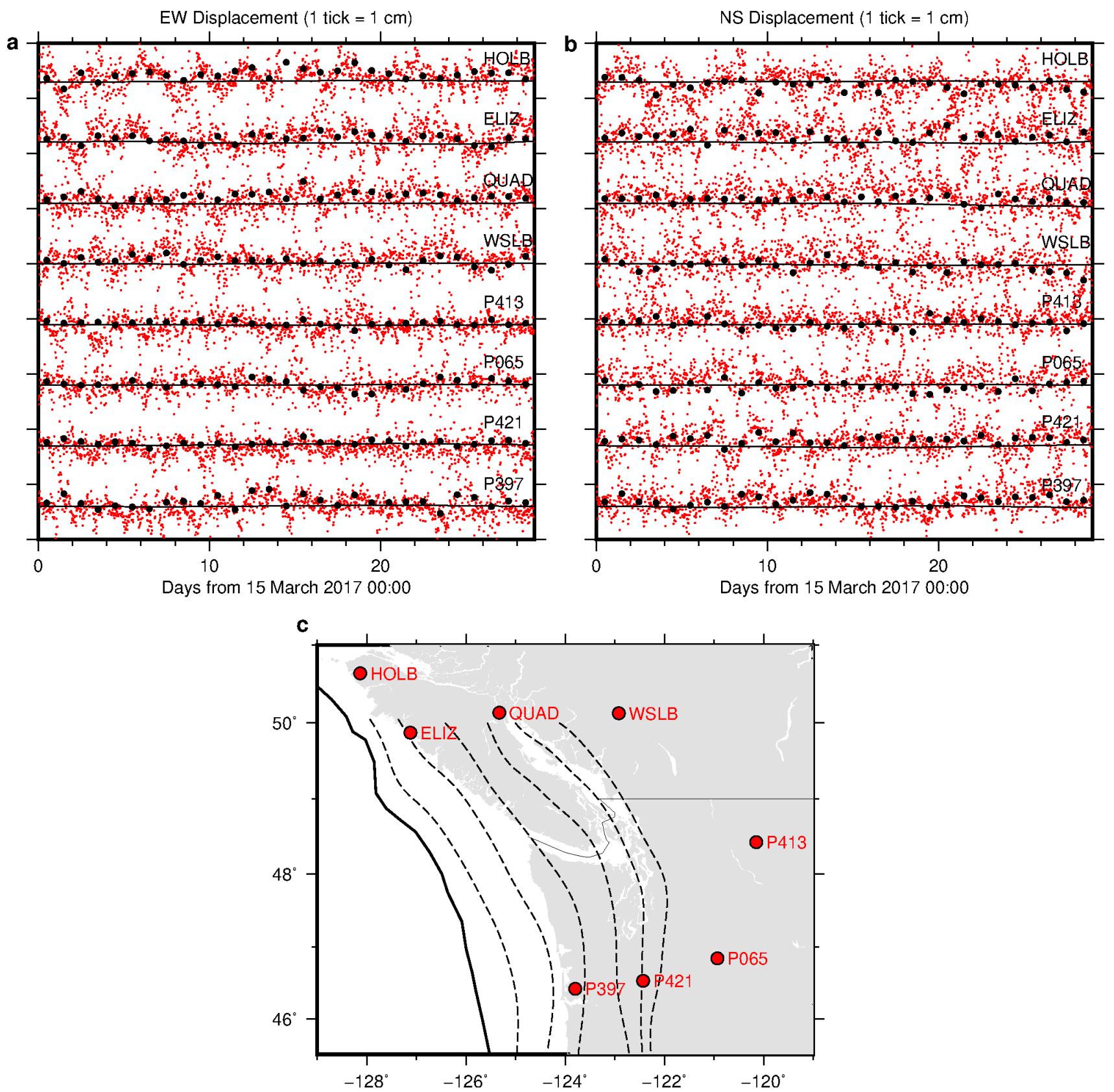

Figure. S2. Kinematic GPS data (30-minute interval) at distant sites from the area of the SSE of interest and fitting by spatiotemporal slip model. $\mathbf{a}-\mathbf{b}$. The red dots indicate the post-processed east (a) and north (b) components of kinematic GPS positions, which are further corrected for whole network translation estimated in slip inversion (see Method). The overlying black dots indicate daily static east component which were corrected for whole network translation estimated in the daily slip inversion (see Method). The overlying black solid lines indicate the predicted motion due to the fault slip. c. Site location. The red circles indicate the site location of the time series in $\mathbf{a}-\mathbf{b}$. 


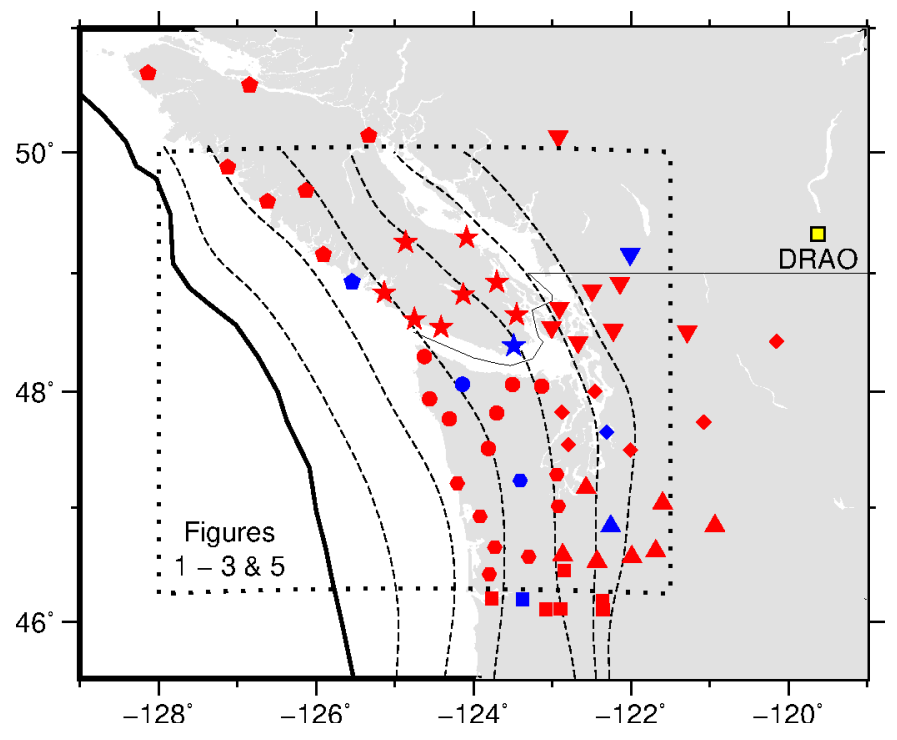

Figure. S3. Group of kinematic sites. Sites in each of the eight groups are indicated by different symbols (red and blue). The blue symbols indicate the reference sites used for kinematic GPS analysis of the sites in each group (red). The yellow square indicates DRAO, the reference site of the entire network. The box with dots indicates the area shown in Figures $1-3$ and 5. 

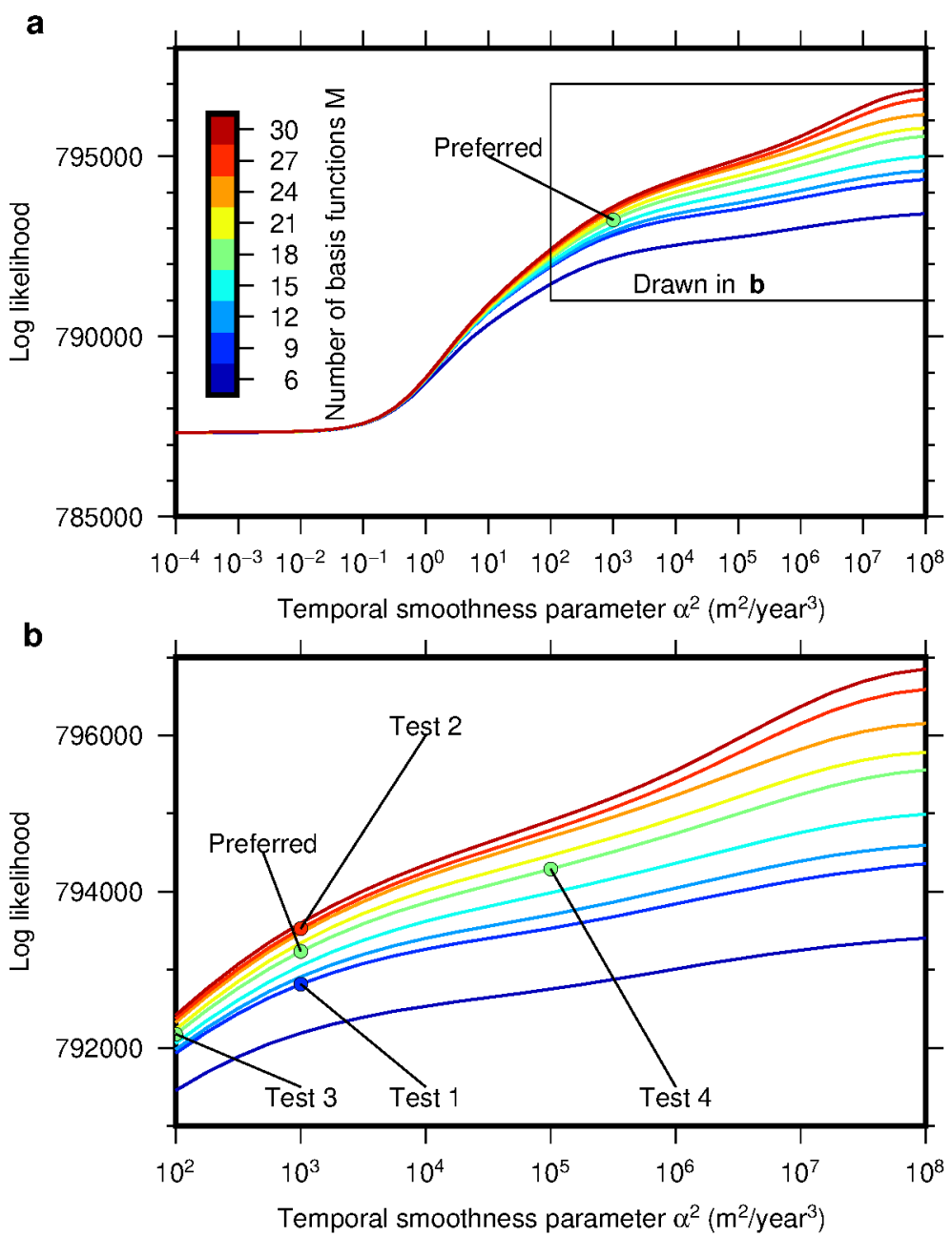

Figure. S4. Diagram of log-likelihood with various hyperparameters. a. The coloured solid lines indicate log-likelihood values with different temporal roughness parameters $\alpha^{2}$. Each colour indicates the number of basis functions, $M$. The box with the solid line indicates the region of $\mathbf{b}$. The green dots indicate the preferred model as labelled. b. Zoom-in plot of log-likelihood curves with a large $\alpha^{2}$. The coloured dots indicate the preferred (Figures 3a, 3c, 3e, 4 and 5) and test models (Figure S5) as labelled. 

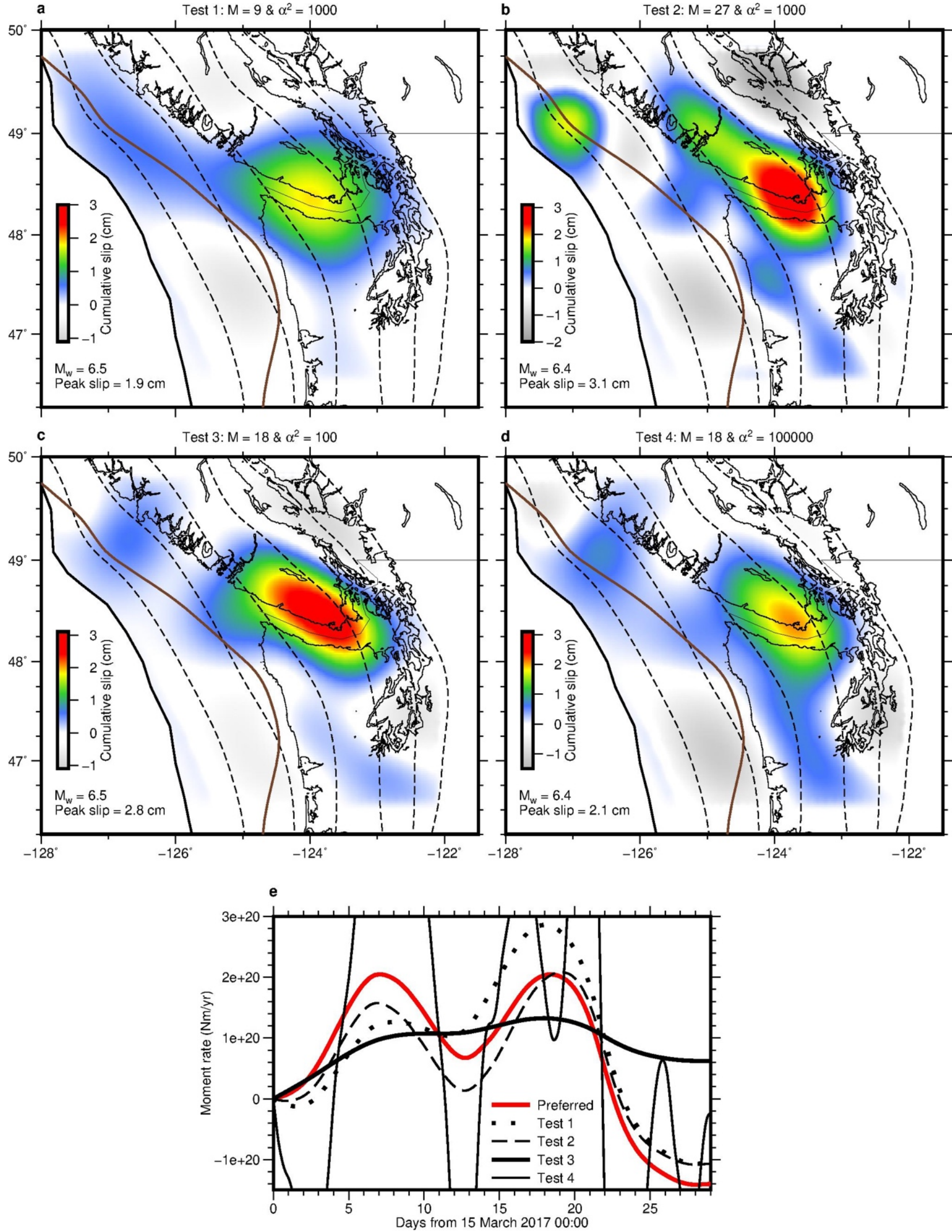

Figure. S5. Test models with different hyperparameters for spatiotemporal smoothness of slip. $\mathbf{a}-\mathbf{d}$. Cumulative slip with different numbers of basis functions $M$ and temporal smoothness parameter $\alpha^{2}$ in $\mathrm{m}^{2} /$ year $^{3}$ as labelled. e. Moment rate history of preferred and test models. Curves with different line types indicate different models, as shown in the legend. The curve for test 4 (thinner solid black line) was saturated. 


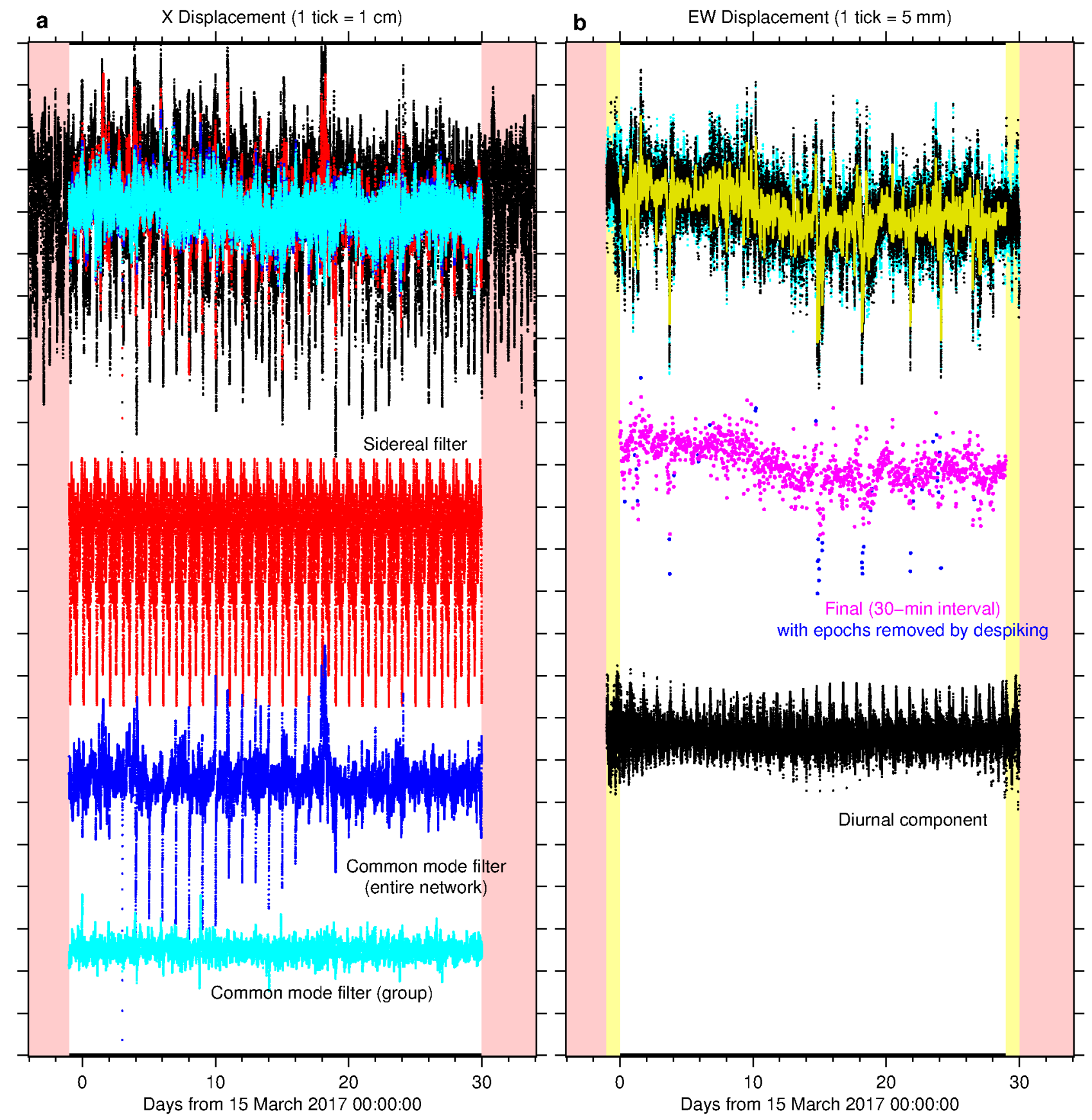

Figure S6. Example of post-processing procedure of kinematic GPS position time series at a 30second interval. a. Displacement in the X-direction in the geocentric coordinate system at ALBH. The top time series indicate the original (black), sidereal-filtered (red), entire-network-commonmode-filtered (blue), and the each-group-common-mode-filtered (light blue) position time series. The filters used for these steps were plotted with the corresponding colours as labelled. The light red background highlights the data period at both ends which are used to construct the sidereal filter. $\mathbf{b}$. Displacement in the EW direction at the ALBH. The top time series indicate the common-modefiltered (light blue), diurnal-component-removed (black), and low-pass filtered (yellow) position time series. The middle blue and purple time series indicate the decimated position time series with an interval of $30 \mathrm{~min}$, from which the epochs indicated in blue were omitted by despiking to obtain the final position time series (purple). The bottom black time series indicates the estimated diurnal components. The yellow background highlights the data period at both ends which are used to taper. 
a
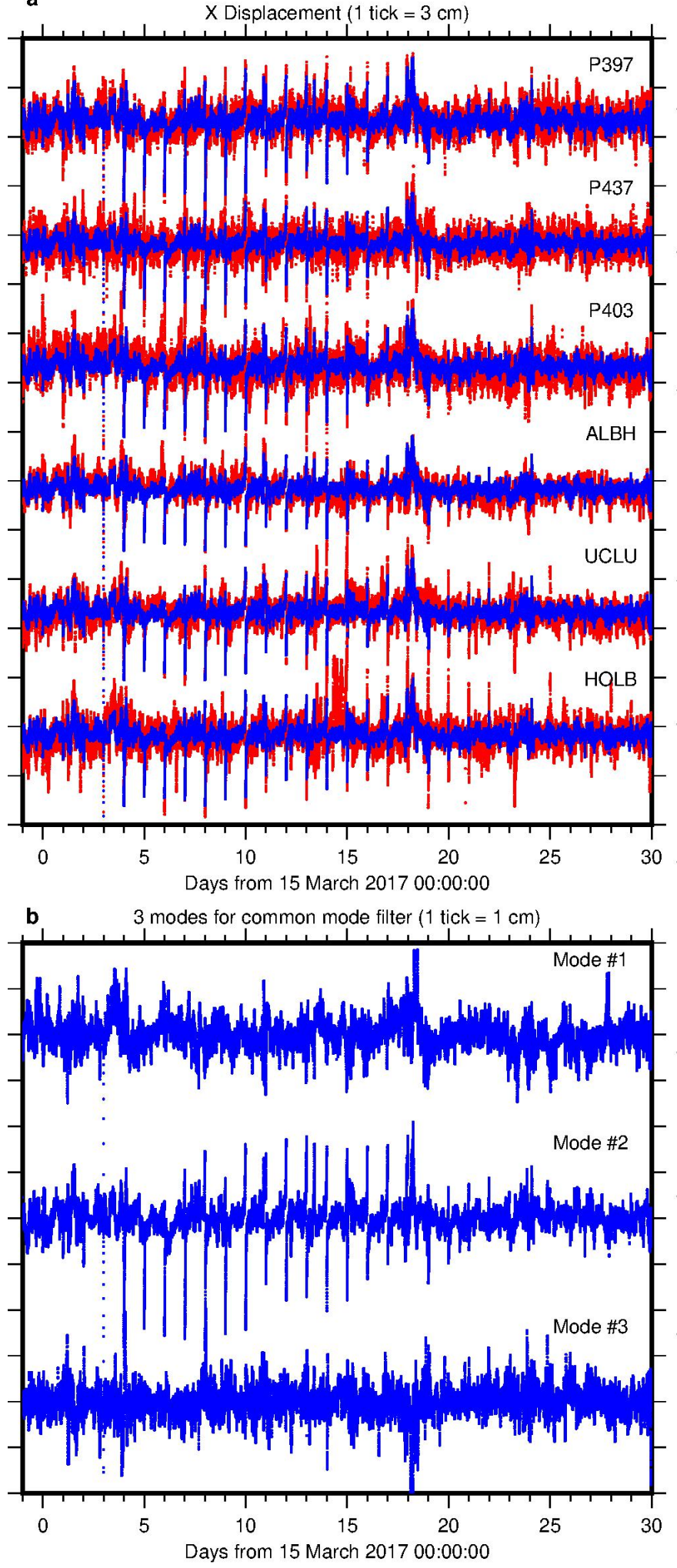

C
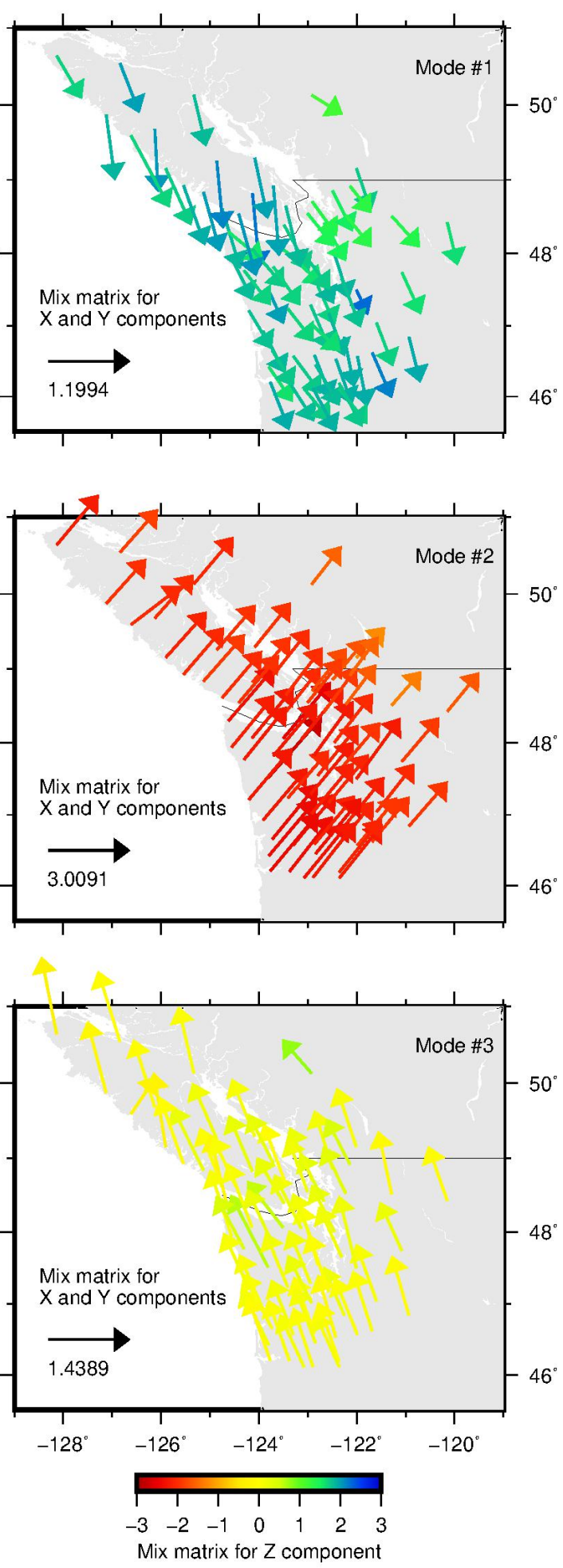

Figure S7. ICA-based common-mode filtering for the entire network a. Examples of post-siderealfiltered position time series in the X-direction in the geocentric coordinate system at selected sites as labelled (red) with common-mode filter estimated at each site (blue). Site locations are shown in Figures S1 and S2. b. Three independent components (modes) representing the common-mode error estimated by the ICA. c. Mix matrix (spatial pattern) for the three modes shown in B. East and north components of the vectors indicate $\mathrm{X}$ and $\mathrm{Y}$ components in the geocentric coordinate system of the mix matrix at each site, respectively, with their $\mathrm{Z}$ components color-coded. 


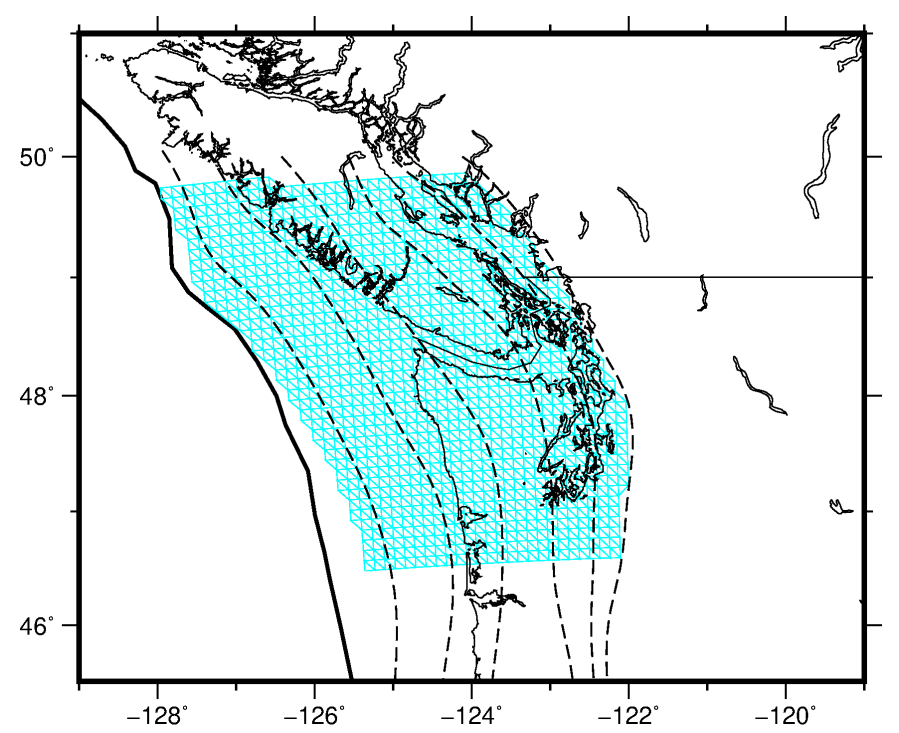

Figure S8. Fault configuration. The light blue triangles indicate the subfaults used to generate the elastic Green's functions. 

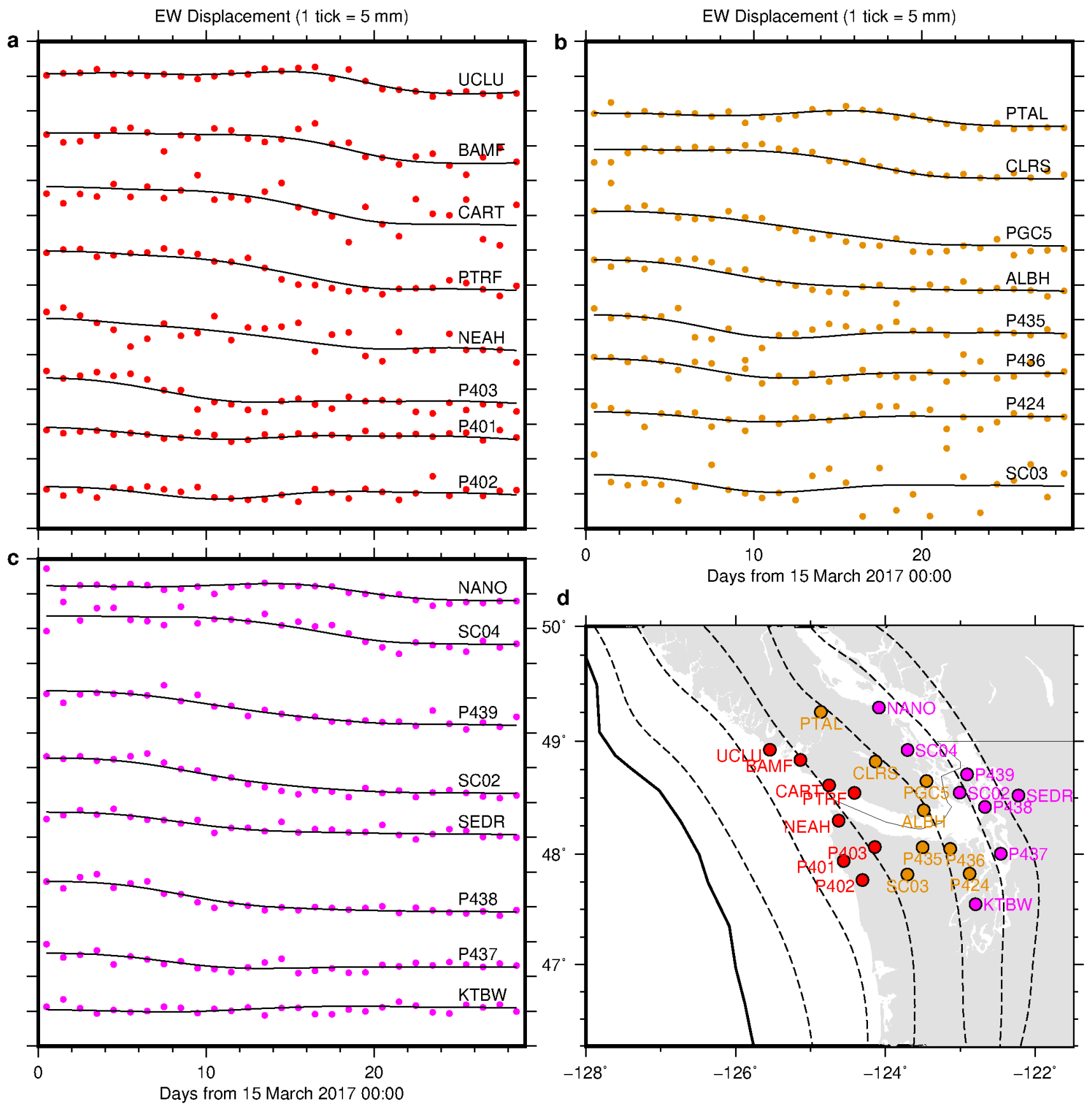

Figure. S9. Daily GPS data and fitting by spatiotemporal slip model. a - c. The coloured dots indicate east component of daily GPS positions, further corrected for whole network translation estimated in the daily slip inversion (Figure 3b, 3d and 3f; see Method). The daily solutions happen to be unavailable at CART and SC03, so we used the kinematic coordinates at every noon instead. The overlying black solid lines indicate the predicted motion due to the fault slip. d. Site location. The coloured circles indicate the site location of the time series with the same colour in $\mathbf{a}-\mathbf{c}$. 

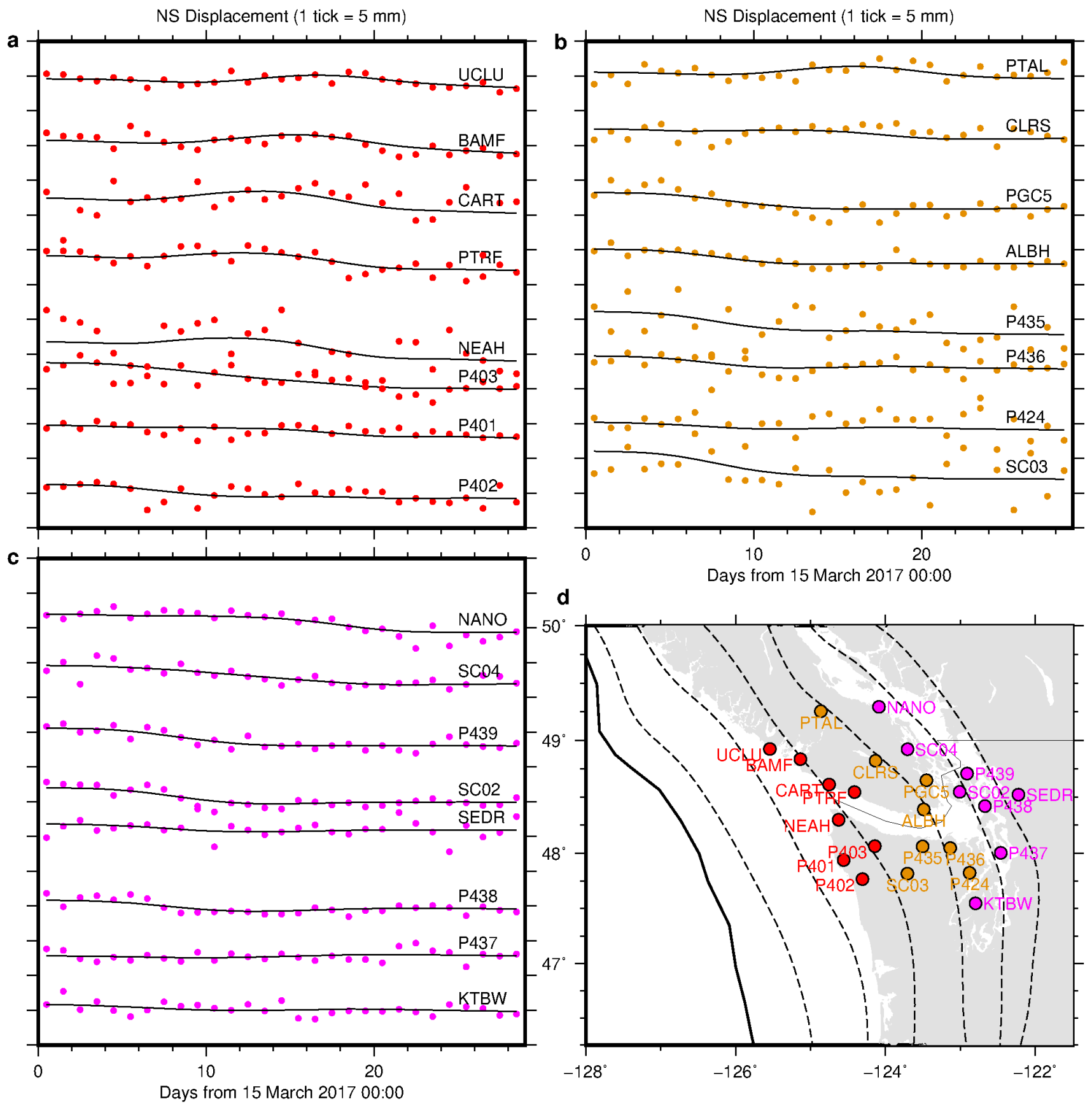

Figure. S10. Daily GPS data and fitting by spatiotemporal slip model. a $-\mathbf{c}$. The coloured dots indicate north component of daily GPS positions, further corrected for whole network translation estimated in the daily slip inversion (Figure 3b, 3d and 3f; see Method). The daily solutions happen to be unavailable at CART and SC03, so we used the kinematic coordinates at every noon instead. The overlying black solid lines indicate the predicted motion due to the fault slip. d. Site location. The coloured circles indicate the site location of the time series with the same colour in $\mathbf{a}-\mathbf{c}$. 
a

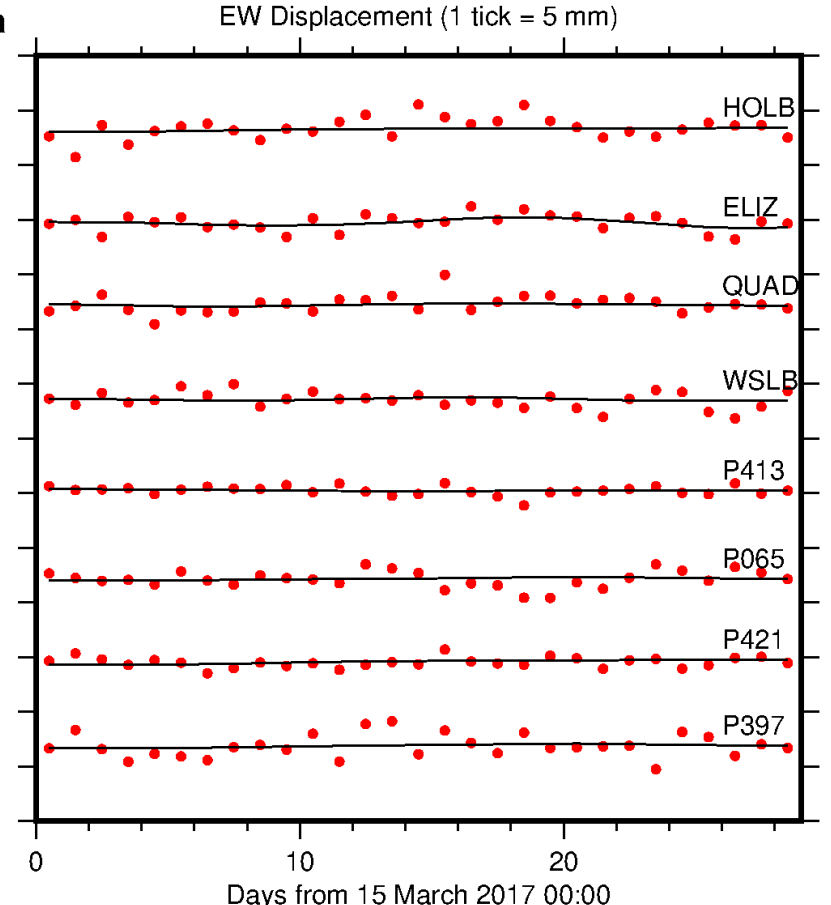

b

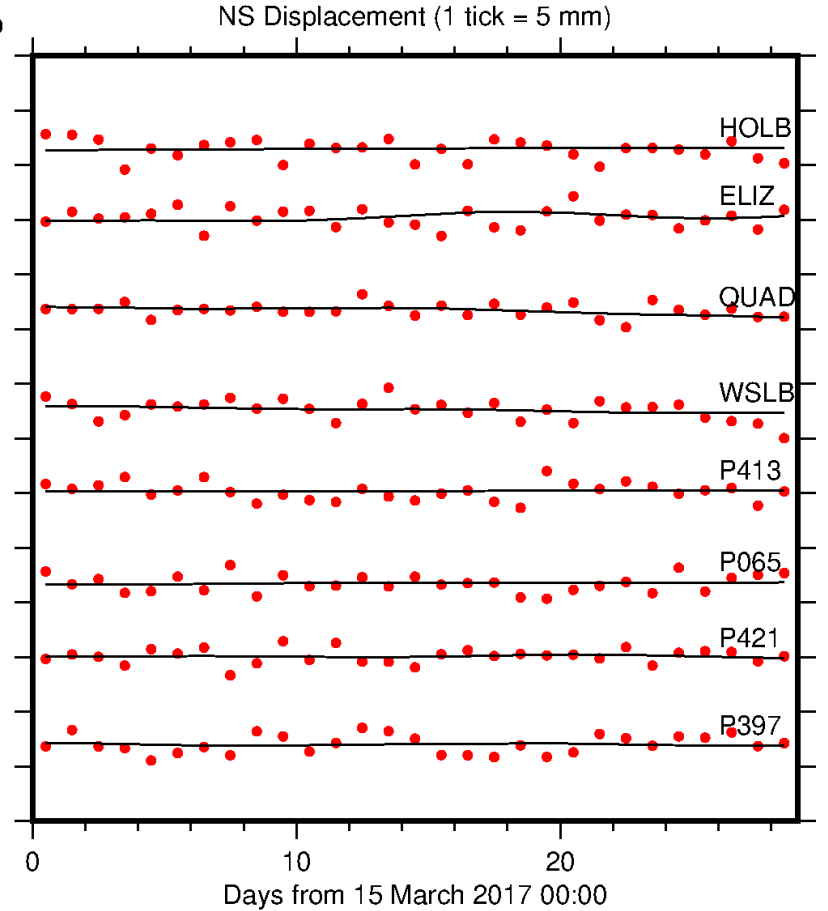

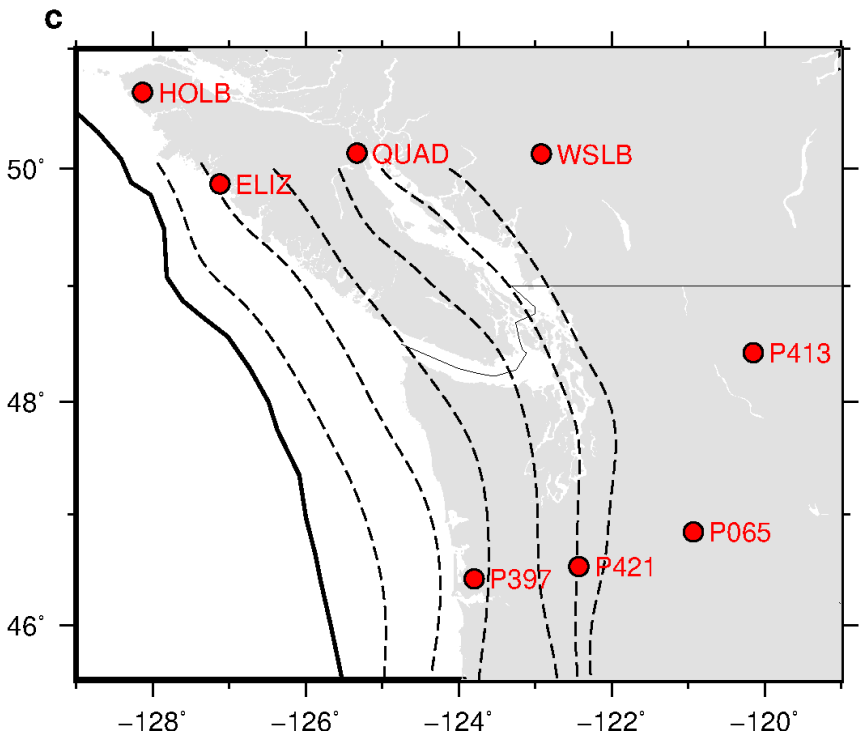

Figure. S11. Daily GPS data at distant sites from the area of the SSE of interest and fitting by spatiotemporal slip model. a - b. The red dots indicate east (a) and north (b) components of daily GPS positions, which are further corrected for whole network translation estimated in slip inversion (Figure 3b, 3d and 3f; see Method). The overlying black solid lines indicate the predicted motion due to the fault slip. c. Site location. The red circles indicate the site location of the time series in $\mathbf{a}-\mathbf{b}$. 


\section{Video S1.}

Slip evolution over the entire period at a 30-minute interval. See Figure 3a for the legends.

\section{Video S2.}

Slip rate evolution over the entire period at a 30-minute interval. The purple dots indicate tremor epicentres during each 30-minute bin. See Figure 3a for other legends.

\section{Video S3.}

Daily slip evolution over the entire period obtained from the inversion of daily GPS data. See Figure $3 \mathrm{~b}$ for the legends.

\section{Video S4.}

Daily slip rate evolution over the entire period obtained from the inversion of daily GPS data. The purple dots indicate tremor epicentres during a daily bin. See Figure $3 \mathrm{~b}$ for other legends. 